\title{
Anisotropic problem with non-local boundary con- ditions and measure data
}

\author{
A. KaBORÉ \\ S. OuAro iD \\ Laboratoire de Mathematiques et \\ Informatiques (LAMI), UFR. Sciences \\ Exactes et Appliquées, Université Joseph \\ KI-ZERBO, 03 BP 7021 Ouaga 03, \\ Ouagadougou, Burkina Faso. \\ kaboreadama59@yahoo.fr; \\ ouaro@yahoo.fr
}

\begin{abstract}
We study a nonlinear anisotropic elliptic problem with nonlocal boundary conditions and measure data. We prove an existence and uniqueness result of entropy solution.

\section{RESUMEN}

Estudiamos un problema elíptico nolineal anisotrópico con condiciones de borde no-locales y data de medida. Probamos un resultado de existencia y unicidad de la solución de entropía.
\end{abstract}

Keywords and Phrases: Entropy solution, non-local boundary conditions, Leray-Lions operator, bounded Radon diffuse measure, Marcinkiewicz spaces.

2020 AMS Mathematics Subject Classification: 35J05, 35J25, 35J60, 35J66.

\section{(cc) BY-NC}

Accepted: 06 January, 2021 Received: 13 November, 2019
(C)2021 A. Kaboré et al. This open access article is licensed under a Creative Commons Attribution-NonCommercial 4.0 International License. 


\section{Introduction and assumptions}

Let $\Omega$ be a bounded domain in $\mathbb{R}^{N}(N \geq 3)$ such that $\partial \Omega$ is Lipschitz and $\partial \Omega=\Gamma_{D} \cup \Gamma_{N e}$ with $\Gamma_{D} \cap \Gamma_{N e}=\emptyset$. Our aim is to study the following problem.

$$
P(\rho, \mu, d) \begin{cases}-\sum_{i=1}^{N} \frac{\partial}{\partial x_{i}} a_{i}\left(x, \frac{\partial}{\partial x_{i}} u\right)+|u|^{p_{M}(x)-2} u=\mu & \text { in } \Omega \\ u=0 & \text { on } \Gamma_{D} \\ \rho(u)+\sum_{i=1}^{N} \int_{\Gamma_{N e}} a_{i}\left(x, \frac{\partial}{\partial x_{i}} u\right) \eta_{i}=d \\ u \equiv \text { constant } & \\ \text { on } \Gamma_{N e},\end{cases}
$$

where the right-hand side $\mu$ is a bounded Radon diffuse measure (that is $\mu$ does not charge the sets of zero $p_{m}($.$) -capacity), \rho: \mathbb{R} \rightarrow \mathbb{R}$ a surjective, continuous and non-decreasing function, with $\rho(0)=0, d \in \mathbb{R}$ and $\eta_{i}, i \in\{1, \ldots, N\}$ are the components of the outer normal unit vector.

For any $\Omega \subset \mathbb{R}^{N}$, we set

$$
C_{+}(\bar{\Omega})=\left\{h \in C(\bar{\Omega}): \inf _{x \in \Omega} h(x)>1\right\}
$$

and we denote

$$
h^{+}=\sup _{x \in \Omega} h(x), \quad h^{-}=\inf _{x \in \Omega} h(x) .
$$

For the exponents, $\vec{p}():. \bar{\Omega} \rightarrow \mathbb{R}^{N}, \vec{p}()=.\left(p_{1}(),. \ldots, p_{N}().\right)$ with $p_{i} \in C_{+}(\bar{\Omega})$ for every $i \in\{1, \ldots, N\}$ and for all $x \in \bar{\Omega}$. We put $p_{M}(x)=\max \left\{p_{1}(x), \ldots, p_{N}(x)\right\}$ and $p_{m}(x)=\min \left\{p_{1}(x), \ldots, p_{N}(x)\right\}$.

We assume that for $i=1, \ldots, N$, the function $a_{i}: \Omega \times \mathbb{R} \rightarrow \mathbb{R}$ is Carathéodory and satisfies the following conditions.

- $\left(H_{1}\right): a_{i}(x, \xi)$ is the continuous derivative with respect to $\xi$ of the mapping $A_{i}=A_{i}(x, \xi)$, that is, $a_{i}(x, \xi)=\frac{\partial}{\partial \xi} A_{i}(x, \xi)$ such that the following equality holds.

$$
A_{i}(x, 0)=0
$$

for almost every $x \in \Omega$.

- $\left(H_{2}\right)$ : There exists a positive constant $C_{1}$ such that

$$
\left|a_{i}(x, \xi)\right| \leq C_{1}\left(j_{i}(x)+|\xi|^{p_{i}(x)-1}\right)
$$

for almost every $x \in \Omega$ and for every $\xi \in \mathbb{R}$, where $j_{i}$ is a non-negative function in $L^{p_{i}^{\prime}(.)}(\Omega)$, with $\frac{1}{p_{i}(x)}+\frac{1}{p_{i}^{\prime}(x)}=1$. 
- $\left(H_{3}\right)$ : there exists a positive constant $C_{2}$ such that

$$
\left(a_{i}(x, \xi)-a_{i}(x, \eta)\right) \cdot(\xi-\eta) \geq \begin{cases}C_{2}|\xi-\eta|^{p_{i}(x)} & \text { if }|\xi-\eta| \geq 1, \\ C_{2}|\xi-\eta|^{p_{i}^{-}} & \text {if }|\xi-\eta|<1,\end{cases}
$$

for almost every $x \in \Omega$ and for every $\xi, \eta \in \mathbb{R}$, with $\xi \neq \eta$.

- $\left(H_{4}\right)$ : For almost every $x \in \Omega$ and for every $\xi \in \mathbb{R}$,

$$
|\xi|^{p_{i}(x)} \leq a_{i}(x, \xi) \cdot \xi \leq p_{i}(x) A_{i}(x, \xi)
$$

- $\left(H_{5}\right)$ : The variable exponents $p_{i}():. \bar{\Omega} \rightarrow[2, N)$ are continuous functions for all $i=1, \ldots, N$ such that

$$
\frac{\bar{p}(N-1)}{N(\bar{p}-1)}<p_{i}^{-}<\frac{\bar{p}(N-1)}{N-\bar{p}}, \sum_{i=1}^{N} \frac{1}{p_{i}^{-}}>1 \text { and } \frac{p_{i}^{+}-p_{i}^{-}-1}{p_{i}^{-}}<\frac{\bar{p}-N}{\bar{p}(N-1)},
$$

where $\frac{1}{\bar{p}}=\frac{1}{N} \sum_{i=1}^{N} \frac{1}{p_{i}^{-}}$.

As examples under assumptions $\left(H_{1}\right)-\left(H_{5}\right)$, we can give the following.

(1) Set $A_{i}(x, \xi)=\left(\frac{1}{p_{i}(x)}\right)|\xi|^{p_{i}(x)}$ and $a_{i}(x, \xi)=|\xi|^{p_{i}(x)-2} \xi$, where $2 \leq p_{i}(x)<N$.

(2) $A_{i}(x, \xi)=\left(\frac{1}{p_{i}(x)}\right)\left(\left(1+|\xi|^{2}\right)^{\frac{p_{i}(x)}{2}}-1\right)$ and $a_{i}(x, \xi)=\left(1+|\xi|^{2}\right)^{\frac{p_{i}(x)-2}{2}} \xi$, where $2 \leq p_{i}(x)<N$.

We put for all $x \in \partial \Omega$,

$$
p^{\partial}(x)= \begin{cases}\frac{(N-1) p(x)}{N-p(x)} & \text { if } p(x)<N \\ \infty & \text { if } p(x) \geq N .\end{cases}
$$

We introduce the numbers

$$
q=\frac{N(\bar{p}-1)}{N-1}, q^{*}=\frac{N q}{N-q}=\frac{N(\bar{p}-1)}{N-\bar{p}} .
$$

We denote by $\mathcal{M}_{b}(\Omega)$ the space of bounded Radon measure in $\Omega$, equipped with its standard norm $\|\cdot\|_{\mathcal{M}_{b}(\Omega)}$. Note that, if $u$ belongs to $\mathcal{M}_{b}(\Omega)$, then $|\mu|(\Omega)$ (the total variation of $\mu$ ) is a bounded positive measure on $\Omega$.

Given $\mu \in \mathcal{M}_{b}(\Omega)$, we say that $\mu$ is diffuse with respect to the capacity $W_{0}^{1, p(.)}(\Omega)(p($.$) -capacity$ for short) if $\mu(A)=0$, for every set $A$ such that $\operatorname{Cap}_{p(.)}(A, \Omega)=0$.

For every $A \subset \Omega$, we denote

$$
S_{p(.)}(A)=\left\{u \in W_{0}^{1, p(\cdot)}(\Omega) \cap C_{0}(\Omega): u=1 \text { on } A, u \geq 0 \text { on } \Omega\right\} .
$$


The $p($.$) -capacity of every subset A$ with respect to $\Omega$ is defined by

$$
\operatorname{Cap}_{p(.)}(A, \Omega)=\inf _{u \in S_{p(.)}(A)}\left\{\int_{\Omega}|\nabla u|^{p(x)} d x\right\} .
$$

In the case $S_{p(.)}(A)=\emptyset$, we set $\operatorname{Cap}_{p(.)}(A, \Omega)=\infty$.

The set of bounded Radon diffuse measure in the variable exponent setting is denoted by $\mathcal{M}_{b}^{p(.)}(\Omega)$. We use the following result of decomposition of bounded Radon diffuse measure proved by Nyanquini et al. (see [31]).

Theorem 1.1. Let $p():. \bar{\Omega} \rightarrow(1, \infty)$ be a continuous function and $\mu \in \mathcal{M}_{b}(\Omega)$. Then $\mu \in \mathcal{M}_{b}^{p(.)}(\Omega)$ if and only if $\mu \in L^{1}(\Omega)+W^{-1, p^{\prime}(.)}(\Omega)$.

Remark 1.2. Since $\mu \in \mathcal{M}_{b}^{p_{m}(.)}(\Omega)$, the Theorem 1.1 implies that there exist $f \in L^{1}(\Omega)$ and $F \in\left(L^{p_{m}^{\prime}(.)}(\Omega)\right)^{N}$ such that

$$
\mu=f-\operatorname{div} F
$$

where $\frac{1}{p_{m}(x)}+\frac{1}{p_{m}^{\prime}(x)}=1, \forall x \in \Omega$.

The study of nonlinear elliptic equations involving the $p$-Laplace operator is based on the theory of standard Sobolev spaces $W^{m, p}(\Omega)$ in order to find weak solutions. For the nonhomogeneous $p($.$) -Laplace operators, the natural setting for this approach is the use of the variable exponent$ Lebesgue and Sobolev spaces $L^{p(.)}(\Omega)$ and $W^{m, p(.)}(\Omega)$.

Variable exponent Lebesgue spaces appeared in the literature for the first time in a article by Orlicz in 1931. In the 1950's, this study was carred on by Nakano who made the first systematic study of spaces with variable exponent (called modular spaces). Nakano explicitly mentioned variable exponent Lebesgue spaces as an example of more general spaces he considered (see [30], p. 284). Later, the polish mathematicians investigated the modular function spaces (see [29]). Note also that H. Hudzik [18] investigated the variable exponent Sobolev spaces. Variable exponent Lebesgue spaces on the real line have been independently developed by Russian researchers, notably Sharapudinov [40] and Tsenov [42]. The next major step in the investigation of variable exponent Lebesgue and Sobolev spaces was the comprehensive paper by O. Kovacik and J. Rakosnik in the early 90's [23]. This paper established many of basic properties of Lebesgue and Sobolev spaces with variables exponent. Variable Sobolev spaces have been used in the last decades to model various phenomena. In [9], Chen, Levine and Rao proposed a framework for image restoration based on a Laplacian variable exponent. Another application which uses nonhomogeneous Laplace operators is related to the modelling of electrorheological fluids see [38]. The first major discovery in electrorheological fluids was due to Winslow in 1949 (cf. [43]). These fluids have the interesting property that their viscosity depends on the electric field in the fluid. They can raise the viscosity by as much as five orders of magnitude. This phenomenon is known as the Winslow effect. For some technical applications, we refer the readers to the work by Pfeiffer et al [33]. Electrorheological fluids have been used in robotics and space technology. The experimental research has been done mainly in 
the USA, for instance in NASA laboratories. For more information on properties, modelling and the application of variable exponent spaces to these fluids, we refer to Diening [11], Rajagopal and Ruzicka [35], and Ruzicka [36]. In this paper, the operator involved in (1.1) is more general than the $p($.$) -Laplace operator. Thus, the variable exponent Sobolev space W^{1, p(.)}(\Omega)$ is not adequate to study nonlinear problems of this type. This leads us to seek entropy solutions for problems (1.1) in a more general variable exponent Sobolev space which was introduced for the first time by Mihaillescu et al. [28], see also [34, 26, 27].

The need for such theory comes naturally every time we want to consider materials with inhomogeneities that have different behavior on different space directions. Non-local boundary value problems of various kinds for partial differential equations are of great interest by now in several fields of application. In a typical non-local problem, the partial differential equation (resp. boundary conditions) for an unknown function $u$ at any point in a domain $\Omega$ involves not only the local behavior of $u$ in a neighborhood of that point but also the non-local behavior of $u$ elsewhere in $\Omega$. For example, at any point in $\Omega$ the partial differential equation and/or the boundary conditions may contains integrals of the unknown $u$ over parts of $\Omega$, values of $u$ elsewhere in $D$ or, generally speaking, some non-local operator on $u$. Beside the mathematical interest of nonlocal conditions, it seems that this type of boundary condition appears in petroleum engineering model for well modeling in a $3 D$ stratified petroleum reservoir with arbitrary geometry (see [12] and [15]). A lot of papers ( see [34], [24], [25], [2], [19], [1]) on problems like (1.1) considered cases of generally boundary value condition. In [6], Bonzi et al. studied the following problems.

$$
\begin{cases}-\sum_{i=1}^{N} \frac{\partial}{\partial x_{i}} a_{i}\left(x, \frac{\partial}{\partial x_{i}} u\right)+|u|^{p_{M}(x)-2} u=f & \text { in } \Omega \\ \sum_{i=1}^{N} a_{i}\left(x, \frac{\partial}{\partial x_{i}} u\right) \eta_{i}=-|u|^{r(x)-2} u & \text { on } \partial \Omega\end{cases}
$$

which correspond to the Robin type boundary condition. The authors used minimization techniques used in [8] to prove the existence and uniqueness of entropy solution. By the same techniques, Koné and al. proved the existence and uniqueness of entropy solution for the following problem.

$$
\begin{cases}-\sum_{i=1}^{N} \frac{\partial}{\partial x_{i}} a_{i}\left(x, \frac{\partial}{\partial x_{i}} u\right)+|u|^{p_{M}(x)-2} u=f & \text { in } \Omega \\ \sum_{i=1}^{N} a_{i}\left(x, \frac{\partial}{\partial x_{i}} u\right) \eta_{i}+\lambda u=g & \text { on } \partial \Omega\end{cases}
$$

which correspond to the Fourier type boundary condition.

In a recent paper we studied a nonlinear elliptic anisotropic problem involving non- local conditions. We also considered variable exponent and general maximal monotone graph datum at the boundary 
and proved existence and uniqueness of weak solution to the following problem.

$$
S(\rho, \mu, d)\left\{\begin{array}{ll}
-\sum_{i=1}^{N} \frac{\partial}{\partial x_{i}} a_{i}\left(x, \frac{\partial}{\partial x_{i}} u\right)+|u|^{p_{M}(x)-2} u=f & \text { in } \Omega \\
u=0 & \text { on } \Gamma_{D} \\
\rho(u)+\sum_{i=1}^{N} \int_{\Gamma_{N e}} a_{i}\left(x, \frac{\partial}{\partial x_{i}} u\right) \eta_{i} \ni d \\
u \equiv \text { constant }
\end{array} \quad \text { on } \Gamma_{N e},\right.
$$

where the right-hand side $f \in L^{\infty}(\Omega)$ and $\rho$ a maximal monotone graph on $\mathbb{R}$ such that $D(\rho)=$ $\operatorname{Im}(\rho)=\mathbb{R}$ and $0 \in \rho(0), d \in \mathbb{R}$, by using the technique of monotone operators in Banach spaces (see [21]) and approximation methods. There are two difficulties associated with the study of problem $P(\rho, \mu, d)$. The first is to give a sense to the partial derivative of $u$ which appear in the term $a_{i}\left(x, \frac{\partial}{\partial x_{i}} u\right)$. As $\mu$ is a measure (even if $\mu$ is a integrable function), then we cannot take the partial derivative of $u$ in the usual distribution sense. The idea consists in considering troncatures of the solution $u$ (see [5]). The second difficulty appears with the question of uniqueness of solutons. We obtain existence and uniqueness of a special class of solutions of problem $P(\rho, \mu, d)$ that satisfy an extra condition that we call the entropy condition (see formula (2.9)). An alternative notion of solution which can leads to existence and uniqueness of solution to problem $P(\rho, \mu, d)$ is the notion of renormalized solution. But in this work, we consider the notion of entropy solution.

The paper is organized as follows. Section 2 is devoted to mathematical preliminaries including, among other things, a brief discussion on variable exponent Lebesgue, Sobolev, anisotropic and Marcinkiewicz spaces. In Section 3, we study an approximated problem and in Section 4, we prove by using the results of the Section 3, the existence and uniqueness of entropy solution of problem $P(\rho, \mu, d)$.

\section{Preliminary}

This part is related to anisotropic Lebesgue and Sobolev spaces with variable exponent and some of their properties.

Given a measurable function $p():. \Omega \rightarrow[1, \infty)$. We define the Lebesgue space with variable exponent $L^{p(.)}(\Omega)$ as the set of all measurable functions $u: \Omega \rightarrow \mathbb{R}$ for which the convex modular

$$
\rho_{p(.)}(u):=\int_{\Omega}|u|^{p(x)} d x
$$

is finite.

If the exponent is bounded, i.e, if $p_{+}<\infty$, then the expression

$$
|u|_{p(.)}:=\inf \left\{\lambda>0: \rho_{p(.)}\left(\frac{u}{\lambda}\right) \leq 1\right\}
$$


defines a norm in $L^{p(\cdot)}(\Omega)$, called the Luxembourg norm. The space $\left(L^{p(\cdot)}(\Omega),|\cdot|_{p(.)}\right)$ is a separable Banach space. Then, $L^{p(.)}(\Omega)$ is uniformly convex, hence reflexive and its dual space is isomorphic to $L^{p^{\prime}(.)}(\Omega)$, where $\frac{1}{p(x)}+\frac{1}{p^{\prime}(x)}=1$, for all $x \in \Omega$. We have the following properties (see [13]) on the modular $\rho_{p(.)}$.

If $u, u_{n} \in L^{p(.)}(\Omega)$ and $p_{+}<\infty$, then

$$
\begin{gathered}
|u|_{p(.)}<1 \Rightarrow|u|_{p(.)}^{p^{+}} \leq \rho_{p(.)}(u) \leq|u|_{p(.)}^{p^{-}}, \\
|u|_{p(.)}>1 \Rightarrow|u|_{p(.)}^{p^{-}} \leq \rho_{p(.)}(u) \leq|u|_{p(.)}^{p^{+}}, \\
|u|_{p(.)}<1(=1 ;>1) \Rightarrow \rho_{p(.)}(u)<1(=1 ;>1),
\end{gathered}
$$

and

$$
\left|u_{n}\right|_{p(.)} \rightarrow 0\left(\left|u_{n}\right|_{p(.)} \rightarrow \infty\right) \Leftrightarrow \rho_{p(.)}\left(u_{n}\right) \rightarrow 0\left(\rho_{p(.)}\left(u_{n}\right) \rightarrow \infty\right) .
$$

If in addition, $\left(u_{n}\right)_{n \in \mathbb{N}} \subset L^{p(.)}(\Omega)$, then $\lim _{n \rightarrow \infty}\left|u_{n}-u\right|_{p(.)}=0 \Leftrightarrow \lim _{n \rightarrow \infty} \rho_{p(.)}\left(u_{n}-u\right)=0 \Leftrightarrow$ $\left(u_{n}\right)_{n \in \mathbb{N}}$ converges to $u$ in measure and $\lim _{n \rightarrow \infty} \rho_{p(.)}\left(u_{n}\right)=\rho_{p(.)}(u)$.

We introduce the definition of the isotropic Sobolev space with variable exponent,

$$
W^{1, p(\cdot)}(\Omega):=\left\{u \in L^{p(\cdot)}(\Omega):|\nabla u| \in L^{p(\cdot)}(\Omega)\right\}
$$

which is a Banach space equipped with the norm

$$
\|u\|_{1, p(.)}:=|u|_{p(.)}+|\nabla u|_{p(.)} .
$$

Now, we present the anisotropic Sobolev space with variable exponent which is used for the study of $P(\rho, \mu, d)$.

The anisotropic variable exponent Sobolev space $W^{1, \vec{p}(.)}(\Omega)$ is defined as follow.

$$
W^{1, \vec{p}(.)}(\Omega):=\left\{u \in L^{p_{M}(.)}(\Omega): \frac{\partial u}{\partial x_{i}} \in L^{p_{i}(.)}(\Omega) \text {, for all } i \in\{1, \ldots, N\}\right\} .
$$

Endowed with the norm

$$
\|u\|_{\vec{p}(.)}:=|u|_{p_{M}(.)}+\sum_{i=1}^{N}\left|\frac{\partial u}{\partial x_{i}}\right|_{p_{i}(.)},
$$

the space $\left(W^{1, \vec{p}(.)}(\Omega),\|\cdot\|_{\vec{p}(.)}\right)$ is a reflexive Banach space (see [14], Theorem 2.1 and Theorem 2.2). As consequence, we have the following.

Theorem 2.1. (see [14]) Let $\Omega \subset \mathbb{R}^{\mathbb{N}}(N \geq 3)$ be a bounded open set and for all $i \in\{1, \ldots, N\}, p_{i} \in$ $L^{\infty}(\Omega), p_{i}(x) \geq 1$ a.e. in $\Omega$. Then, for any $r \in L^{\infty}(\Omega)$ with $r(x) \geq 1$ a.e. in $\Omega$ such that

$$
\text { ess } \inf _{x \in \Omega}\left(p_{M}(x)-r(x)\right)>0
$$

we have the compact embedding

$$
W^{1, \vec{p}(\cdot)}(\Omega) \hookrightarrow L^{r(\cdot)}(\Omega)
$$


We also need the following trace theorem due to [7].

Theorem 2.2. Let $\Omega \subset \mathbb{R}^{\mathbb{N}}(N \geq 2)$ be a bounded open set with smooth boundary and let $\vec{p}(.) \in$ $C(\bar{\Omega})$ satisfy the condition

$$
1 \leq r(x)<\min _{x \in \partial \Omega}\left\{p_{1}^{\partial}(x), \ldots, p_{N}^{\partial}(x)\right\}, \forall x \in \partial \Omega
$$

Then, there is a compact boundary trace embedding

$$
W^{1, \vec{p}(.)}(\Omega) \hookrightarrow L^{r(\cdot)}(\partial \Omega)
$$

Let us introduce the following notation:

$$
\vec{p}_{-}=\left(p_{1}^{-}, \ldots, p_{N}^{-}\right)
$$

We will use in this paper, the Marcinkiewicz spaces $\mathcal{M}^{q}(\Omega)(1<q<\infty)$ with constant exponent. Note that the Marcinkiewicz spaces $\mathcal{M}^{q(.)}(\Omega)$ in the variable exponent setting was introduced for the first time by Sanchon and Urbano (see [37]).

Marcinkiewicz spaces $\mathcal{M}^{q}(\Omega)(1<q<\infty)$ contain all measurable function $h: \Omega \rightarrow \mathbb{R}$ for which the distribution function

$$
\lambda_{h}(\gamma):=\operatorname{meas}(\{x \in \Omega:|h(x)|>\gamma\}), \gamma \geq 0
$$

satisfies an estimate of the form $\lambda_{h}(\gamma) \leq C \gamma^{-q}$, for some finite constant $C>0$.

The space $\mathcal{M}^{q}(\Omega)$ is a Banach space under the norm

$$
\|h\|_{\mathcal{M}^{q}(\Omega)}^{*}=\sup _{t>0} t^{\frac{1}{q}}\left(\frac{1}{t} \int_{0}^{t} h^{*}(s) d s\right),
$$

where $h^{*}$ denotes the nonincreasing rearrangement of $h$.

$$
h^{*}(t):=\inf \left\{C: \lambda_{h}(\gamma) \leq C \gamma^{-q}, \forall \gamma>0\right\}
$$

which is equivalent to the norm $\|h\|_{\mathcal{M}^{q}(\Omega)}^{*}($ see $[3])$.

We need the following Lemma (see [4], Lemma A-2).

Lemma 2.3. Let $1 \leq q<p<\infty$. Then, for every measurable function $u$ on $\Omega$,

(i) $\frac{(p-1)^{p}}{p^{p+1}}\|u\|_{\mathcal{M}^{p}(\Omega)}^{p} \leq \sup _{\lambda>0}\left\{\lambda^{p} \operatorname{meas}[x \in \Omega:|u|>\lambda]\right\} \leq\|u\|_{\mathcal{M}^{p}(\Omega)}^{p}$. Moreover,

(ii) $\int_{K}|u|^{q} d x \leq \frac{p}{p-q}\left(\frac{p}{q}\right)^{\frac{q}{p}}\|u\|_{\mathcal{M}^{p}(\Omega)}^{q}(\text { meas }(K))^{\frac{p-q}{p}}$, for every measurable subset $K \subset \Omega$.

In particular, $\mathcal{M}^{p}(\Omega) \subset L_{\text {loc }}^{q}(\Omega)$, with continuous embedding and $u \in \mathcal{M}^{p}(\Omega)$ implies $|u|^{q} \in$ $\mathcal{M}^{\frac{p}{q}}(\Omega)$. 
The following result is due to Troisi (see [39]).

Theorem 2.4. Let $p_{1}, \ldots, p_{N} \in[1, \infty), \vec{p}=\left(p_{1}, \ldots, p_{N}\right) ; g \in W^{1, \vec{p}}(\Omega)$, and let

$$
\begin{cases}q=\bar{p}^{*} & \text { if } \quad \bar{p}^{*}<N \\ q \in[1, \infty) & \text { if } \quad \bar{p}^{*} \geq N\end{cases}
$$

where $p^{*}=\frac{N}{\sum_{i=1}^{N} \frac{1}{p_{i}}-1}, \sum_{i=1}^{N} \frac{1}{p_{i}}>1$ and $\bar{p}^{*}=\frac{N \bar{p}}{N-\bar{p}}$.

Then, there exists a constant $C>0$ depending on $N, p_{1}, \ldots, p_{N}$ if $\bar{p}<N$ and also on $q$ and meas $(\Omega)$ if $\bar{p} \geq N$ such that

$$
\|g\|_{L^{q}(\Omega)} \leq c \prod_{i=1}^{N}\left[\|g\|_{L^{p_{M}(\Omega)}}+\left\|\frac{\partial g}{\partial x_{i}}\right\|_{L^{p_{i}(\Omega)}}\right]^{\frac{1}{N}}
$$

where $p_{M}=\max \left\{p_{1}, \ldots, p_{N}\right\}$ and $\frac{1}{\bar{p}}=\frac{1}{N} \sum_{i=1}^{N} \frac{1}{p_{i}}$. In particular, if $u \in W_{0}^{1, \vec{p}}(\Omega)$, we have

$$
\|g\|_{L^{q}(\Omega)} \leq c \prod_{i=1}^{N}\left[\left\|\frac{\partial g}{\partial x_{i}}\right\|_{L^{p_{i}(\Omega)}}\right]^{\frac{1}{N}} .
$$

In the sequel, we consider the following spaces.

$$
W_{D}^{1, \vec{p}(.)}(\Omega)=\left\{\xi \in W^{1, \vec{p}(\cdot)}(\Omega): \xi=0 \text { on } \Gamma_{D}\right\}
$$

and

$$
\begin{gathered}
W_{N e}^{1, \vec{p}(.)}(\Omega)=\left\{\xi \in W_{D}^{1, \vec{p}(.)}(\Omega): \xi \equiv \text { constant on } \Gamma_{N e}\right\} . \\
\mathcal{T}_{D}^{1, \vec{p}(.)}(\Omega)=\left\{\xi \text { measurable on } \Omega \text { such that } \forall k>0, T_{k}(\xi) \in W_{D}^{1, \vec{p}(.)}(\Omega)\right\}
\end{gathered}
$$

and

$$
\mathcal{T}_{N e}^{1, \vec{p}(.)}(\Omega)=\left\{\xi \text { measurable on } \Omega \text { such that } \forall k>0, T_{k}(\xi) \in W_{N e}^{1, \vec{p}(.)}(\Omega)\right\},
$$

where $T_{k}$ is a truncation function defined by

$$
T_{k}(s)= \begin{cases}k & \text { if } s>k \\ s & \text { if }|s| \leq k \\ -k & \text { if } s<-k\end{cases}
$$

For any $v \in W_{N e}^{1, \vec{p}(.)}(\Omega)$, we set $v_{N}=v_{N e}:=\left.v\right|_{\Gamma_{N e}}$.

Definition 2.5. A measurable function $u: \Omega \rightarrow \mathbb{R}$ is an entropy solution of $P(\rho, \mu, d)$ if $u \in$ $\mathcal{T}_{N e}^{1, \vec{p}(.)}(\Omega)$ and for every $k>0$,

$$
\left\{\begin{array}{l}
\int_{\Omega}\left(\sum_{i=1}^{N} a_{i}\left(x, \frac{\partial}{\partial x_{i}} u\right) \frac{\partial}{\partial x_{i}} T_{k}(u-\xi)\right) d x+\int_{\Omega}|u|^{p_{M}(x)-2} u T_{k}(u-\xi) d x \leq \\
\int_{\Omega} T_{k}(u-\xi) d \mu+\left(d-\rho\left(u_{N e}\right)\right) T_{k}\left(u_{N e}-\xi\right),
\end{array}\right.
$$

for all $\xi \in W_{N e}^{1, \vec{p}(.)}(\Omega) \cap L^{\infty}(\Omega)$. 
Our main result in this paper is the following theorem.

Theorem 2.6. Assume $\left(H_{1}\right)-\left(H_{5}\right)$. Then for any $(\mu, d) \in \mathcal{M}_{b}^{p_{m}(\cdot)}(\Omega) \times \mathbb{R}$, the problem $P(\rho, \mu, d)$ admits a unique entropy solution $u$.

\section{The approximated problem corresponding to $P(\rho, \mu, d)$}

We define a new bounded domain $\tilde{\Omega}$ in $\mathbb{R}^{N}$ as follow.

We fix $\theta>0$ and we set $\tilde{\Omega}=\Omega \cup\left\{x \in \mathbb{R}^{N} / \operatorname{dist}\left(x, \Gamma_{N e}\right)<\theta\right\}$. Then, $\partial \tilde{\Omega}=\Gamma_{D} \cup \tilde{\Gamma}_{N e}$ is Lipschitz with $\Gamma_{D} \cap \tilde{\Gamma}_{N e}=\emptyset$.

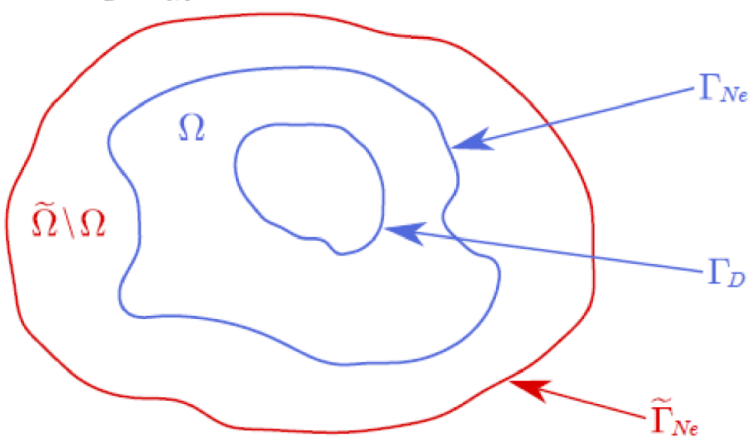

Figure 1: Domains representation

Let us consider $\tilde{a}_{i}(x, \xi)$ (to be defined later) Carathéodory and satisfying (1.4), (1.5), (1.6) and (1.7), for all $x \in \tilde{\Omega}$.

We also consider a function $\tilde{d}$ in $L^{1}\left(\tilde{\Gamma}_{N e}\right)$ such that

$$
\int_{\tilde{\Gamma}_{N e}} \tilde{d} d \sigma=d
$$

For any $\epsilon>0$, we set $\mu_{\epsilon}=f_{\epsilon}-\operatorname{div} F$, where $f_{\epsilon}=T_{\frac{1}{\epsilon}}(f) \in L^{\infty}(\Omega)$. Note that $f_{\epsilon} \rightarrow f$ as $\epsilon \rightarrow 0$ in $L^{1}(\Omega)$ and $\left\|f_{\epsilon}\right\|_{1} \leq\|f\|_{1}$.

We set $\tilde{\mu}_{\epsilon}=f_{\epsilon} \chi_{\Omega}-\operatorname{div} F \chi_{\Omega}, \tilde{d}_{\epsilon}=T_{\frac{1}{\epsilon}}(\tilde{d})$ and we consider the problem

$$
P\left(\tilde{\rho}, \tilde{\mu}_{\epsilon}, \tilde{d}_{\epsilon}\right) \begin{cases}-\sum_{i=1}^{N} \frac{\partial}{\partial x_{i}} \tilde{a}_{i}\left(x, \frac{\partial}{\partial x_{i}} u_{\epsilon}\right)+\left|u_{\epsilon}\right|^{p_{M}(x)-2} u_{\epsilon} \chi_{\Omega}(x)=\tilde{\mu}_{\epsilon} & \text { in } \tilde{\Omega} \\ u_{\epsilon}=0 & \text { on } \Gamma_{D} \\ \tilde{\rho}\left(u_{\epsilon}\right)+\sum_{i=1}^{N} \tilde{a}_{i}\left(x, \frac{\partial}{\partial x_{i}} u_{\epsilon}\right) \eta_{i}=\tilde{d}_{\epsilon} & \text { on } \tilde{\Gamma}_{N e}\end{cases}
$$

where the function $\tilde{\rho}$ is defined as follow. 
- $\tilde{\rho}(s)=\frac{1}{\left|\tilde{\Gamma}_{N e}\right|} \rho(s)$, where $\left|\tilde{\Gamma}_{N e}\right|$ denotes the Hausdorff measure of $\tilde{\Gamma}_{N e}$.

We obviously have $\forall \epsilon>0, \tilde{d}_{\epsilon} \in L^{\infty}\left(\tilde{\Gamma}_{N e}\right)$.

The following definition gives the notion of solution for the problem $P_{\epsilon}\left(\tilde{\rho}, \tilde{\mu}_{\epsilon}, \tilde{d}_{\epsilon}\right)$.

Definition 3.1. A measurable function $u_{\epsilon}: \tilde{\Omega} \rightarrow \mathbb{R}$ is a solution to problem $P_{\epsilon}\left(\tilde{\rho}, \tilde{\mu}_{\epsilon}, \tilde{d}_{\epsilon}\right)$ if $u_{\epsilon} \in$ $W_{D}^{1, \vec{p}(\cdot)}(\tilde{\Omega})$ and

$$
\int_{\tilde{\Omega}} \sum_{i=1}^{N} \tilde{a}_{i}\left(x, \frac{\partial}{\partial x_{i}} u_{\epsilon}\right) \frac{\partial}{\partial x_{i}} \tilde{\xi} d x+\int_{\Omega}\left|u_{\epsilon}\right|^{p_{M}(x)-2} u_{\epsilon} \tilde{\xi} d x=\int_{\Omega} f_{\epsilon} \tilde{\xi} d x+\int_{\Omega} F \cdot \nabla \tilde{\xi}+\int_{\tilde{\Gamma}_{N e}}\left(\tilde{d}_{\epsilon}-\tilde{\rho}\left(u_{\epsilon}\right)\right) \tilde{\xi} d \sigma
$$

for any $\tilde{\xi} \in W_{D}^{1, \vec{p}(.)}(\tilde{\Omega}) \cap L^{\infty}(\Omega)$.

Theorem 3.2. The problem $P_{\epsilon}\left(\tilde{\rho}, \tilde{\mu}_{\epsilon}, \tilde{d}_{\epsilon}\right)$ admits at least one solution in the sense of Definition 3.1.

Step 1: Approximated problem we study an existence result to the following problem. For any $k>0$ we consider

$$
P_{\epsilon, k}\left(\tilde{\rho}, \tilde{\mu}_{\epsilon}, \tilde{d}_{\epsilon}\right) \begin{cases}-\sum_{i=1}^{N} \frac{\partial}{\partial x_{i}} \tilde{a}_{i}\left(x, \frac{\partial}{\partial x_{i}} u_{\epsilon, k}\right)+T_{k}\left(b\left(u_{\epsilon, k}\right)\right) \chi_{\Omega}(x)=\tilde{\mu}_{\epsilon} & \text { in } \tilde{\Omega} \\ u_{\epsilon, k}=0 & \text { on } \Gamma_{D} \\ T_{k}\left(\tilde{\rho}\left(u_{\epsilon, k}\right)\right)+\sum_{i=1}^{N} \tilde{a}_{i}\left(x, \frac{\partial}{\partial x_{i}} u_{\epsilon, k}\right) \eta_{i}=\tilde{d}_{\epsilon} & \text { on } \tilde{\Gamma}_{N e},\end{cases}
$$

where $b(u)=|u|^{p_{M}(x)-2} u$.

We have to prove that $P_{\epsilon, k}\left(\tilde{\rho}, \tilde{\mu}_{\epsilon}, \tilde{d}_{\epsilon}\right)$ admits at least one solution in the following sense.

$$
\left\{\begin{array}{l}
u_{\epsilon, k} \in W_{D}^{1, \vec{p}(.)}(\tilde{\Omega}) \text { and for all } \tilde{\xi} \in W_{D}^{1, \vec{p}(.)}(\tilde{\Omega}), \\
\int_{\tilde{\Omega}} \sum_{i=1}^{N} \tilde{a}_{i}\left(x, \frac{\partial}{\partial x_{i}} u_{\epsilon, k}\right) \frac{\partial}{\partial x_{i}} \tilde{\xi} d x+\int_{\Omega} T_{k}\left(b\left(u_{\epsilon, k}\right)\right) \tilde{\xi} d x=\int_{\Omega} \tilde{\xi} d \mu_{\epsilon}+\int_{\tilde{\Gamma}_{N e}}\left(\tilde{d}_{\epsilon}-T_{k}\left(\tilde{\rho}\left(u_{\epsilon, k}\right)\right)\right) \tilde{\xi} d \sigma .
\end{array}\right.
$$

For any $k>0$, let us introduce the operator $\Lambda_{k}: W_{D}^{1, \vec{p}(\cdot)}(\tilde{\Omega}) \rightarrow\left(W_{D}^{1, \vec{p}(.)}(\tilde{\Omega})\right)^{\prime}$ such that for any $(u, v) \in W_{D}^{1, \vec{p}(\cdot)}(\tilde{\Omega}) \times W_{D}^{1, \vec{p}(\cdot)}(\tilde{\Omega})$,

$$
\left\langle\Lambda_{k}(u), v\right\rangle=\int_{\tilde{\Omega}}\left(\sum_{i=1}^{N} \tilde{a}_{i}\left(x, \frac{\partial}{\partial x_{i}} u\right) \frac{\partial}{\partial x_{i}} v\right) d x+\int_{\Omega} T_{k}(b(u)) v d x+\int_{\tilde{\Gamma}_{N e}} T_{k}(\tilde{\rho}(u)) v d \sigma .
$$

We need to prove that for any $k>0$, the operator $\Lambda_{k}$ is bounded, coercive, of type $M$ and therefore, surjective.

(i) Boundedness of $\Lambda_{k}$. Let $(u, v) \in F \times W_{D}^{1, \vec{p}(\cdot)}(\tilde{\Omega})$ with $F$ a bounded subset of $W_{D}^{1, \vec{p}(.)}(\tilde{\Omega})$. 
We have

$$
\left\{\begin{array}{l}
\left|\left\langle\Lambda_{k}(u), v\right\rangle\right| \leq \sum_{i=1}^{N}\left(\int_{\tilde{\Omega}}\left|\tilde{a}_{i}\left(x, \frac{\partial}{\partial x_{i}} u\right)\right|\left|\frac{\partial}{\partial x_{i}} v\right| d x\right)+\int_{\tilde{\Omega}}\left|T_{k}(b(u))\right||v| d x+\int_{\tilde{\Gamma}_{N e}}\left|T_{k}(\tilde{\rho}(u))\right||v| d \sigma \\
=I_{1}+I_{2}+I_{3},
\end{array}\right.
$$

where we denote by $I_{1}, I_{2}$ and $I_{3}$ the three terms on the right hand side of the first inequality. By $\left(H_{2}\right)$ and the Hölder type inequality, we have

$$
\left\{\begin{array}{l}
I_{1} \leq C_{1} \sum_{i=1}^{N}\left(\int_{\tilde{\Omega}}\left|j_{i}(x)\right|\left|\frac{\partial}{\partial x_{i}} v\right| d x+\int_{\tilde{\Omega}}\left|\frac{\partial}{\partial x_{i}} u\right|^{p_{i}(x)-1}\left|\frac{\partial}{\partial x_{i}} v\right| d x\right) \\
\leq C_{1} \sum_{i=1}^{N}\left(\frac{1}{p_{i}^{\prime-}}+\frac{1}{p_{i}^{-}}\right)\left|j_{i}\right|_{p_{i}^{\prime}(.)}\left|\frac{\partial}{\partial x_{i}} v\right|_{p_{i}(.)}+\left.\left.\sum_{i=1}^{N}\left(\frac{1}{p_{i}^{\prime-}}+\frac{1}{p_{i}^{-}}\right)|| \frac{\partial}{\partial x_{i}} u\right|^{p_{i}(x)-1}\right|_{p_{i}^{\prime}(.)}\left|\frac{\partial}{\partial x_{i}} v\right|_{p_{i}(.)} .
\end{array}\right.
$$

As $u \in F, \forall i \in\{1, \ldots, N\}$, there exists a constant $M>0$ such that

$$
\left.\left.\sum_{i=1}^{N}|| \frac{\partial}{\partial x_{i}} u\right|^{p_{i}(x)-1}\right|_{p_{i}^{\prime}(.)}<M
$$

so

$$
\left.\left.|| \frac{\partial}{\partial x_{i}} u\right|^{p_{i}(x)-1}\right|_{p_{i}^{\prime}(.)}<M, \forall i \in\{1, \ldots, N\}
$$

Let $C_{4}=\max _{i=1, \ldots, N}\left\{\left.\left.|| \frac{\partial}{\partial x_{i}} u\right|^{p_{i}(x)-1}\right|_{p_{i}^{\prime}(.)}\right\}$.

As $j_{i} \in L^{p_{i}^{\prime}(\cdot)}(\tilde{\Omega})$, we have

$$
I_{1} \leq C_{5}\left(C_{1}, p_{i}^{-},\left(p_{i}^{\prime}\right)^{-}, C_{3}\left(j_{i}\right)\right) \sum_{i=1}^{N}\left|\frac{\partial}{\partial x_{i}} v\right|_{p_{i}(.)}+C_{6}\left(C_{1}, p_{i}^{-},\left(p_{i}^{\prime}\right)^{-}, C_{4}\right) \sum_{i=1}^{N}\left|\frac{\partial}{\partial x_{i}} v\right|_{p_{i}(.)} .
$$

It is easy to see that

$$
I_{2} \leq k \int_{\tilde{\Omega}}|v| d x
$$

Using Theorem 2.1, we have

$$
\|v\|_{L^{1}(\tilde{\Omega})} \leq C_{7}\|v\|_{W_{D}^{1, \vec{p}(\cdot)}(\tilde{\Omega})}
$$

So,

$$
I_{2} \leq k C_{7}\|v\|_{W_{D}^{1, \vec{p}(\cdot)}(\tilde{\Omega})} \cdot
$$

Similarly, by using Theorem 2.2, we have

$$
I_{3} \leq k C_{8}\|v\|_{W_{D}^{1, \vec{p}(.)}(\tilde{\Omega})} \square
$$

Therefore, $\Lambda_{k}$ maps bounded subsets of $W_{D}^{1, \vec{p}(.)}(\tilde{\Omega})$ into bounded subsets of $\left(W_{D}^{1, \vec{p}(.)}(\tilde{\Omega})\right)^{\prime}$. Thus, $\Lambda_{k}$ is bounded on $W_{D}^{1, \vec{p}(.)}(\tilde{\Omega})$. 
(ii) Coerciveness of $\Lambda_{k}$. We have to show that for any $k>0, \frac{\left\langle\Lambda_{k}(u), u\right\rangle}{\|u\|_{W_{D}^{1, \vec{p}(\cdot)}(\tilde{\Omega})}} \rightarrow \infty$ as $\|u\|_{W_{D}^{1, \vec{p}(\cdot)}(\tilde{\Omega})} \rightarrow \infty$.

For any $u \in W_{D}^{1, \vec{p}(\cdot)}(\tilde{\Omega})$, we have

$$
\left\langle\Lambda_{k}(u), u\right\rangle=\langle\Lambda(u), u\rangle+\int_{\Omega} T_{k}(b(u)) u d x+\int_{\tilde{\Gamma}_{N e}} T_{k}(\tilde{\rho}(u)) u d \sigma,
$$

where $\langle\Lambda(u), u\rangle=\sum_{i=1}^{N}\left(\int_{\tilde{\Omega}} \tilde{a}_{i}\left(x, \frac{\partial}{\partial x_{i}} u\right) \frac{\partial}{\partial x_{i}} u d x\right)$.

The last two terms on the right-hand side of (3.7) are non-negative by the monotonicity of $T_{k}, b$ and $\tilde{\rho}$. We can assert that

$$
\left\{\begin{array}{l}
\left\langle\Lambda_{k}(u), u\right\rangle \geq\langle\Lambda(u), u\rangle \\
\geq \frac{1}{N^{p_{m}^{-}-1}}\|u\|_{W_{D}^{1, \vec{p}(.)}(\tilde{\Omega})}^{p^{-}}-N
\end{array}\right.
$$

Indeed, since $\int_{\tilde{\Omega}}\left|T_{k}(b(u))\right||u| d x+\int_{\tilde{\Gamma}_{N e}}\left|T_{k}(\tilde{\rho}(u))\right||u| d \sigma \geq 0$, for all $u \in W_{D}^{1, \vec{p}(.)}(\tilde{\Omega})$, we have

$$
\left\langle\Lambda_{k}(u), u\right\rangle \geq\langle\Lambda(u), u\rangle .
$$

So,

$$
\left\langle\Lambda_{k}(u), u\right\rangle \geq \sum_{i=1}^{N}\left(\int_{\tilde{\Omega}} \tilde{a}_{i}\left(x, \frac{\partial}{\partial x_{i}} u\right) \frac{\partial}{\partial x_{i}} u d x\right) \geq \sum_{i=1}^{N}\left(\int_{\tilde{\Omega}}\left|\frac{\partial}{\partial x_{i}} u\right|^{p_{i}(x)} d x\right) .
$$

We make the following notations:

$$
\mathcal{I}=\left\{i \in\{1, \ldots, N\}:\left|\frac{\partial}{\partial x_{i}} u\right|_{p_{i}(.)} \leq 1\right\} \text { and } \mathcal{J}=\left\{i \in\{1, \ldots, N\}:\left|\frac{\partial}{\partial x_{i}} u\right|_{p_{i}(.)}>1\right\} .
$$

We have

$$
\begin{aligned}
\left\langle\Lambda_{k}(u), u\right\rangle & \geq \sum_{i \in \mathcal{I}}\left(\int_{\tilde{\Omega}}\left|\frac{\partial}{\partial x_{i}} u\right|^{p_{i}(x)} d x\right)+\sum_{i \in \mathcal{J}}\left(\int_{\tilde{\Omega}}\left|\frac{\partial}{\partial x_{i}} u\right|^{p_{i}(x)} d x\right) \\
& \geq \sum_{i \in \mathcal{I}}\left(\left|\frac{\partial}{\partial x_{i}} u\right|_{p_{i}(.)}^{p_{i}^{+}}\right)+\sum_{i \in \mathcal{J}}\left(\left|\frac{\partial}{\partial x_{i}} u\right|_{p_{i}(.)}^{p_{i}^{-}}\right) \\
& \geq \sum_{i \in \mathcal{J}}\left(\left|\frac{\partial}{\partial x_{i}} u\right|_{p_{i}(.)}^{p_{i}^{-}}\right) \\
& \geq \sum_{i \in \mathcal{J}}\left(\left|\frac{\partial}{\partial x_{i}} u\right|_{p_{i}(.)}^{p_{m}^{-}}\right) \\
& \geq \sum_{i=1}^{N}\left(\left|\frac{\partial}{\partial x_{i}} u\right|_{p_{i}(.)}^{p_{m}^{-}}\right)-\sum_{i \in \mathcal{I}}\left(\left|\frac{\partial}{\partial x_{i}} u\right|_{p_{i}(.)}^{p_{m}^{-}}\right) \\
& \geq \sum_{i=1}^{N}\left(\left|\frac{\partial}{\partial x_{i}} u\right|_{p_{i}(.)}^{p_{m}^{-}}\right)-N .
\end{aligned}
$$


We now use Jensen's inequality on the convex function $Z: \mathbb{R}^{+} \rightarrow \mathbb{R}^{+}, Z(t)=t^{p_{m}^{-}}, p_{m}^{-}>1$ to get

$$
\left\{\begin{array}{l}
\left\langle\Lambda_{k}(u), u\right\rangle \geq\langle\Lambda(u), u\rangle \\
\geq \frac{1}{N^{p_{m}^{-}-1}}\|u\|_{W_{D}^{1, \vec{p}(.)}(\tilde{\Omega})}^{p^{-}}-N .
\end{array}\right.
$$

Hence, $\Lambda_{k}$ is coercive (as $p_{m}^{-}>1$ ).

\section{(iii) The operator $\Lambda_{k}$ is of type $M$.}

Lemma 3.3. (cf [41]) Let $\mathcal{A}$ and $\mathcal{B}$ be two operators. If $\mathcal{A}$ is of type $M$ and $\mathcal{B}$ is monotone and weakly continuous, then $\mathcal{A}+\mathcal{B}$ is of type $M$.

Now, we set $\langle\mathcal{A} u, v\rangle:=\langle\Lambda(u), v\rangle$ and $\left\langle\mathcal{B}_{k} u, v\right\rangle:=\int_{\Omega} T_{k}(b(u)) v d x+\int_{\tilde{\Gamma}_{N e}} T_{k}(\tilde{\rho}(u)) v d \sigma$.

Then, for every $k>0$, we have $\Lambda_{k}=\mathcal{A}+\mathcal{B}_{k}$. We now have to show that for every $k>0$, $\mathcal{B}_{k}$ is monotone and weakly continuous, because it is well-known that $\mathcal{A}$ is of type $M$. For the monotonicity of $\mathcal{B}_{k}$, we have to show that

$$
\left\langle\mathcal{B}_{k} u-\mathcal{B}_{k} v, u-v\right\rangle \geq 0 \text { for all }(u, v) \in W_{D}^{1, \vec{p}(.)}(\tilde{\Omega}) \times W_{D}^{1, \vec{p}(.)}(\tilde{\Omega}) .
$$

We have

$$
\begin{aligned}
\left\langle\mathcal{B}_{k} u-\mathcal{B}_{k} v, u-v\right\rangle & =\int_{\Omega}\left(T_{k}(b(u))-T_{k}(b(v))\right)(u-v) d x \\
& +\int_{\tilde{\Gamma}_{N e}}\left(T_{k}(\tilde{\rho}(u))-T_{k}(\tilde{\rho}(v))\right)(u-v) d \sigma .
\end{aligned}
$$

From the monotonicity of $b, \tilde{\rho}$ and the map $T_{k}$, we conclude that

$$
\left\langle\mathcal{B}_{k} u-\mathcal{B}_{k} v, u-v\right\rangle \geq 0 .
$$

We need now to prove that for each $k>0$ the operator $\mathcal{B}_{k}$ is weakly continuous, that is, for all sequences $\left(u_{n}\right)_{n \in \mathbb{N}} \subset W_{D}^{1, \vec{p}(.)}(\tilde{\Omega})$ such that $u_{n} \rightarrow u$ in $W_{D}^{1, \vec{p}(.)}(\tilde{\Omega})$, we have $\mathcal{B}_{k} u_{n} \rightarrow \mathcal{B}_{k} u$ as $n \rightarrow \infty$. For all $\phi \in W_{D}^{1, \vec{p}(.)}(\tilde{\Omega})$, we have

$$
\left\langle\mathcal{B}_{k} u_{n}, \phi\right\rangle:=\int_{\Omega} T_{k}\left(b\left(u_{n}\right)\right) \phi d x+\int_{\tilde{\Gamma}_{N e}} T_{k}\left(\tilde{\rho}\left(u_{n}\right)\right) \phi d \sigma .
$$

Passing to the limit in (3.9) as $n$ goes to $\infty$ and using the Lebesgue dominated convergence theorem, since $u_{n} \rightarrow u$ in $W_{D}^{1, \vec{p}(.)}(\tilde{\Omega})$; up to a subsequence, we have $u_{n} \rightarrow u$ in $L^{1}(\tilde{\Omega})$ and a.e. in $\tilde{\Omega}$. As $\left|T_{k}\left(b\left(u_{n}\right)\right) \phi\right| \leq k|\phi|$ and $\phi \in W_{D}^{1, \vec{p}(.)}(\tilde{\Omega}) \hookrightarrow L^{1}(\tilde{\Omega})$, for the first term on the right-hand side of (3.9), we obtain

$$
\lim _{n \rightarrow \infty} \int_{\Omega} T_{k}\left(b\left(u_{n}\right)\right) \phi d x=\int_{\Omega} T_{k}(b(u)) \phi d x .
$$

Furthermore, since $u_{n} \rightarrow u$ in $W_{D}^{1, \vec{p}(.)}(\tilde{\Omega})$; up to a subsequence, we have $u_{n} \rightarrow u$ in $L^{1}(\partial \tilde{\Omega})$ and a.e. on $\partial \tilde{\Omega}$. As $\left|T_{k}\left(\tilde{\rho}\left(u_{n}\right)\right) \phi\right| \leq k|\phi|$ and $\phi \in W_{D}^{1, \vec{p}(.)}(\tilde{\Omega}) \hookrightarrow L^{1}(\partial \tilde{\Omega})$, we deduce by the Lebesgue dominated convergence theorem that

$$
\lim _{n \rightarrow \infty} \int_{\tilde{\Gamma}_{N e}} T_{k}\left(\tilde{\rho}\left(u_{n}\right)\right) \phi d x=\int_{\tilde{\Gamma}_{N e}} T_{k}(\tilde{\rho}(u)) \phi d x .
$$


From (3.10) and (3.11) we conclude that for every $k>0, \mathcal{B}_{k}\left(u_{n}\right) \rightarrow \mathcal{B}_{k}(u)$ as $n \rightarrow \infty$.

The operator $\mathcal{A}$ is type $M$ and as $\mathcal{B}_{k}$ is monotone and weakly continuous, thanks to Lemma 3.3, we conclude that the operator $\Lambda_{k}$ is of type $M$. Then for any $L \in\left(W_{D}^{1, \vec{p}(\cdot)}(\tilde{\Omega})\right)^{\prime}$, there exists $u_{\epsilon, k} \in W_{D}^{1, \vec{p}(.)}(\tilde{\Omega})$, such that $\Lambda_{k}\left(u_{\epsilon, k}\right)=L$.

We now consider $L \in\left(W_{D}^{1, \vec{p}(\cdot)}(\tilde{\Omega})\right)^{\prime}$ defined by $L(v)=\int_{\Omega} v d \mu_{\epsilon}+\int_{\tilde{\Gamma}_{N e}} \tilde{d}_{\epsilon} v d \sigma$, for $v \in W_{D}^{1, \vec{p}(.)}(\tilde{\Omega})$ and we obtain (3.5) $\square$

\section{Step 2: A priori estimates}

Lemma 3.4. Let $u_{\epsilon, k}$ a solution of $P_{\epsilon, k}\left(\tilde{\rho}, \tilde{\mu}_{\epsilon}, \tilde{d}_{\epsilon}\right)$. Then

$$
\left\{\begin{array}{l}
\left|\tilde{\rho}\left(u_{\epsilon, k}\right)\right| \leq k_{1}:=\max \left\{\left\|\tilde{d}_{\epsilon}\right\|_{\infty},\left(\tilde{\rho}_{\epsilon} \circ b^{-1}\right)\left(\left\|\mu_{\epsilon}\right\|_{\infty}\right)\right\} \text { a.e. on } \tilde{\Gamma}_{N e} \\
\left|b\left(u_{\epsilon, k}\right)\right| \leq k_{2}:=\max \left\{\mid \mu_{\epsilon} \|_{\infty} ;\left(b \circ \rho_{0}^{-1}\right)\left(\left|\tilde{\Gamma}_{N e}\right|\left\|\tilde{d}_{\epsilon}\right\|_{\infty}\right)\right\} \text { a.e. in } \Omega .
\end{array}\right.
$$

Proof. For any $\tau>0$, let us introduce the function $H_{\tau}: \mathbb{R} \rightarrow \mathbb{R}$ by

$$
H_{\tau}(s)= \begin{cases}0 & \text { if } s<0 \\ \frac{s}{\tau} & \text { if } 0 \leq s \leq \tau \\ 1 & \text { if } s>\tau\end{cases}
$$

In (3.5) we set $\tilde{\xi}=H_{\tau}\left(u_{\epsilon, k}-M\right)$, where $M>0$ is to be fixed later. We get

$$
\left\{\begin{array}{l}
\int_{\tilde{\Omega}} \sum_{i=1}^{N} \tilde{a}_{i}\left(x, \frac{\partial}{\partial x_{i}} u_{\epsilon, k}\right) \frac{\partial}{\partial x_{i}} H_{\tau}\left(u_{\epsilon, k}-M\right) d x+\int_{\Omega} T_{k}\left(b\left(u_{\epsilon, k}\right)\right) H_{\tau}\left(u_{\epsilon, k}-M\right) d x= \\
\int_{\Omega} H_{\tau}\left(u_{\epsilon, k}-M\right) d \mu_{\epsilon}+\int_{\tilde{\Gamma}_{N e}}\left(\tilde{d}_{\epsilon}-T_{k}\left(\tilde{\rho}\left(u_{\epsilon}, k\right)\right)\right) H_{\tau}\left(u_{\epsilon, k}-M\right) d \sigma
\end{array}\right.
$$

The first term in (3.13) is non-negative. Indeed,

$$
\int_{\tilde{\Omega}} \sum_{i=1}^{N} \tilde{a}_{i}\left(x, \frac{\partial}{\partial x_{i}} u_{\epsilon, k}\right) \frac{\partial}{\partial x_{i}} H_{\tau}\left(u_{\epsilon, k}-M\right) d x=\frac{1}{\tau} \int_{\left\{0 \leq u_{\epsilon, k}-M \leq \tau\right\}} \sum_{i=1}^{N} \tilde{a}_{i}\left(x, \frac{\partial}{\partial x_{i}} u_{\epsilon, k}\right) \frac{\partial}{\partial x_{i}} u_{\epsilon, k} d x \geq 0 .
$$

From (3.13) we obtain

$$
\int_{\Omega} T_{k}\left(b\left(u_{\epsilon, k}\right)\right) H_{\tau}\left(u_{\epsilon, k}-M\right) d x \leq \int_{\Omega} H_{\tau}\left(u_{\epsilon, k}-M\right) d \mu_{\epsilon}+\int_{\tilde{\Gamma}_{N e}}\left(\tilde{d}_{\epsilon}-T_{k}\left(\tilde{\rho}\left(u_{\epsilon}, k\right)\right)\right) H_{\tau}\left(u_{\epsilon, k}-M\right) d \sigma .
$$

Then, one has

$$
\left\{\begin{array}{l}
\int_{\Omega}\left(T_{k} b\left(u_{\epsilon, k}\right)-T_{k}(b(M))\right) H_{\tau}\left(u_{\epsilon, k}-M\right) d x+\int_{\tilde{\Gamma}_{N e}}\left(T_{k}\left(\tilde{\rho}\left(u_{\epsilon}, k\right)\right)-T_{k}(\tilde{\rho}(M))\right) H_{\tau}\left(u_{\epsilon, k}-M\right) d x \leq \\
\int_{\Omega}\left(\mu_{\epsilon}-T_{k}(b(M))\right) H_{\tau}\left(u_{\epsilon, k}-M\right) d x+\int_{\tilde{\Gamma}_{N e}}\left(\tilde{d}_{\epsilon}-T_{k}(\tilde{\rho}(M))\right) H_{\tau}\left(u_{\epsilon, k}-M\right) d \sigma .
\end{array}\right.
$$

Letting $\tau$ go to 0 in the inequality above, we get

$$
\left\{\begin{array}{l}
\int_{\Omega}\left(T_{k}\left(b\left(u_{\epsilon, k}\right)\right)-T_{k}(b(M))\right)^{+} d x+\int_{\tilde{\Gamma}_{N e}}\left(T_{k}\left(\tilde{\rho}\left(u_{\epsilon, k}\right)\right)-T_{k}(\tilde{\rho}(M))\right)^{+} d \sigma \leq \\
\int_{\Omega}\left(\mu_{\epsilon}-T_{k}(b(M))\right) \operatorname{sign}_{0}^{+}\left(u_{k}-M\right) d x+\int_{\tilde{\Gamma}_{N e}}\left(\tilde{d}_{\epsilon}-T_{k}(\tilde{\rho}(M))\right) \operatorname{sign}_{0}^{+}\left(u_{\epsilon, k}-M\right) d \sigma .
\end{array}\right.
$$


As $\operatorname{Im}(b)=\operatorname{Im}(\rho)=\mathbb{R}$, we can fix $M=M_{0}=\max \left\{b^{-1}\left(\left\|\mu_{\epsilon}\right\|_{\infty}\right), \rho_{0}^{-1}\left(\left|\tilde{\Gamma}_{N e}\right|\left\|\tilde{d}_{\epsilon}\right\|_{\infty}\right)\right\}$. From the above inequality we obtain

$$
\left\{\begin{array}{l}
\int_{\Omega}\left(T_{k}\left(b\left(u_{\epsilon, k}\right)\right)-T_{k}\left(b\left(M_{0}\right)\right)\right)^{+} d x+\int_{\tilde{\Gamma}_{N e}}\left(T_{k}\left(\tilde{\rho}\left(u_{\epsilon, k}\right)-T_{k}\left(\tilde{\rho}\left(M_{0}\right)\right)\right)^{+} d \sigma \leq\right. \\
\int_{\Omega}\left(\mu_{\epsilon}-T_{k}\left(\left\|\mu_{\epsilon}\right\|_{\infty}\right)\right) \operatorname{sign}_{0}^{+}\left(u_{\epsilon, k}-M_{0}\right) d x+\int_{\tilde{\Gamma}_{N e}}\left(\tilde{d}-T_{k}\left(\left\|\tilde{d}_{\epsilon}\right\|_{\infty}\right)\right) \operatorname{sign}_{0}^{+}\left(u_{\epsilon, k}-M_{0}\right) d \sigma .
\end{array}\right.
$$

For $k>k_{0}:=\max \left\{\left\|\mu_{\epsilon}\right\|,\left\|\tilde{d}_{\epsilon}\right\|_{\infty}\right\}$, it follows that

$$
\int_{\Omega}\left(T_{k}\left(b\left(u_{\epsilon, k}\right)\right)-T_{k}\left(b\left(M_{0}\right)\right)\right)^{+} d x+\int_{\tilde{\Gamma}_{N e}}\left(T_{k}\left(\tilde{\rho}\left(u_{\epsilon, k}\right)\right)-T_{k}\left(\tilde{\rho}\left(M_{0}\right)\right)\right)^{+} d \sigma \leq 0 .
$$

From (3.14), we deduce that

$$
\left\{\begin{array}{l}
T_{k}\left(\tilde{\rho}\left(u_{\epsilon, k}\right)\right) \leq T_{k}\left(\tilde{\rho}\left(M_{0}\right)\right) \text { a.e. on } \tilde{\Gamma}_{N e}, \\
T_{k}\left(b\left(u_{\epsilon, k}\right)\right) \leq T_{k}\left(b\left(M_{0}\right)\right) \text { a.e. in } \Omega .
\end{array}\right.
$$

From (3.15), we deduce that for every $k>k_{1}:=\max \left\{\left\|\tilde{d}_{\epsilon}\right\|_{\infty},\left\|\mu_{\epsilon}\right\|_{\infty}, b\left(M_{0}\right), \tilde{\rho}\left(M_{0}\right)\right\}$,

$$
\tilde{\rho}\left(u_{\epsilon, k}\right) \leq \tilde{\rho}\left(M_{0}\right) \text { a.e. on } \tilde{\Gamma}_{N e}
$$

and

$$
b\left(u_{\epsilon, k}\right) \leq b\left(M_{0}\right) \text { a.e. in } \Omega .
$$

Note that with the choice of $M_{0}$ and the fact that $D(\rho)=D(b)=\mathbb{R}$, for every $k>k_{1}:=$ $\max \left\{\left\|\tilde{d}_{\epsilon}\right\|_{\infty},\left\|\mu_{\epsilon}\right\|_{\infty}, b\left(M_{0}\right), \tilde{\rho}\left(M_{0}\right)\right\}$, we have

$$
\left\{\begin{array}{l}
b\left(u_{\epsilon, k}\right) \leq \max \left\{\left\|\mu_{\epsilon}\right\|_{\infty}, b \circ \rho_{0}^{-1}\left(\left|\tilde{\Gamma}_{N e}\right|\left\|\tilde{d}_{\epsilon}\right\|_{\infty}\right)\right\} \text { a.e. in } \Omega \\
\tilde{\rho}\left(u_{\epsilon, k}\right) \leq \max \left\{\left\|\tilde{d}_{\epsilon}\right\|_{\infty},\left(\tilde{\rho} \circ b^{-1}\right)\left(\left\|\mu_{\epsilon}\right\|_{\infty}\right)\right\} \text { a.e. on } \tilde{\Gamma}_{N e} .
\end{array}\right.
$$

We need to show that for any $k$ large enough,

$$
\left\{\begin{array}{l}
b\left(u_{\epsilon, k}\right) \geq-\max \left\{\left\|\mu_{\epsilon}\right\|_{\infty}, b \circ \rho_{0}^{-1}\left(\left|\tilde{\Gamma}_{N e}\right|\left\|\tilde{d}_{\epsilon}\right\|_{\infty}\right)\right\} \text { a.e. in } \Omega \\
\tilde{\rho}\left(u_{\epsilon, k}\right) \geq-\max \left\{\left\|\tilde{d}_{\epsilon}\right\|_{\infty},\left(\tilde{\rho} \circ b^{-1}\right)\left(\left\|\mu_{\epsilon}\right\|_{\infty}\right)\right\} \text { a.e. on } \tilde{\Gamma}_{N e}
\end{array}\right.
$$

It is easy to see that if $\left(u_{\epsilon, k}\right)$ is a solution of $P_{\epsilon, k}\left(\tilde{\rho}, \tilde{\mu}_{\epsilon}, \tilde{d}_{\epsilon}\right)$, then $\left(-u_{\epsilon, k}\right)$ is a solution of

$$
P_{\epsilon, k}\left(\hat{\rho}, \hat{\mu}_{\epsilon}, \hat{d}_{\epsilon}\right) \begin{cases}-\sum_{i=1}^{N} \frac{\partial}{\partial x_{i}} \hat{a}_{i}\left(x, \frac{\partial}{\partial x_{i}} u_{\epsilon, k}\right)+T_{k}\left(\hat{b}\left(u_{\epsilon, k}\right)\right) \chi_{\Omega}(x)=\hat{\mu}_{\epsilon} & \text { in } \tilde{\Omega} \\ u_{\epsilon, k}=0 & \text { on } \Gamma_{D} \\ T_{k}\left(\hat{\rho}\left(u_{\epsilon, k}\right)\right)+\sum_{i=1}^{N} \hat{a}_{i}\left(x, \frac{\partial}{\partial x_{i}} u_{\epsilon, k}\right) \eta_{i}=\hat{d}_{\epsilon} & \text { on } \tilde{\Gamma}_{N e},\end{cases}
$$

where $\hat{a}_{i}(x, \xi)=-\tilde{a}_{i}(x,-\xi), \hat{\rho}(s)=-\tilde{\rho}(-s), \hat{b}(s)=-b(-s), \hat{\mu}_{\epsilon}=-\tilde{\mu}_{\epsilon}$ and $\hat{d}=-\tilde{d}_{\epsilon}$.

Then for every $k>k_{2}:=\max \left\{\left\|\tilde{d}_{\epsilon}\right\|_{\infty},\left\|\mu_{\epsilon}\right\|_{\infty},-b\left(-M_{0}\right),-\tilde{\rho}\left(-M_{0}\right)\right\}$, we have

$$
\left\{\begin{array}{l}
-b\left(u_{\epsilon, k}\right) \leq \max \left\{\left\|\mu_{\epsilon}\right\|_{\infty}, b \circ \rho_{0}^{-1}\left(\left|\tilde{\Gamma}_{N e}\right|\left\|\tilde{d}_{\epsilon}\right\|_{\infty}\right)\right\} \text { a.e. in } \Omega, \\
-\tilde{\rho}\left(u_{\epsilon, k}\right) \leq \max \left\{\left\|\tilde{d}_{\epsilon}\right\|_{\infty},\left(\tilde{\rho} \circ b^{-1}\right)\left(\left\|\mu_{\epsilon}\right\|_{\infty}\right)\right\} \text { a.e. on } \tilde{\Gamma}_{N e},
\end{array}\right.
$$


which implies (3.17).

From (3.16) and (3.17), we deduce (3.12).

Step 3. Convergence Since $u_{\epsilon, k}$ is a solution of $P_{\epsilon, k}\left(\tilde{\rho}, \tilde{\mu}_{\epsilon}, \tilde{d}_{\epsilon}\right)$, thanks to Lemma 3.4 and the fact that $\Omega$ is bounded, we have $\tilde{\rho}\left(u_{\epsilon, k}\right) \in L^{1}\left(\tilde{\Gamma}_{N e}\right)$ and $b\left(u_{\epsilon, k}\right) \in L^{1}(\Omega)$. For $k=1+\max \left(k_{1}, k_{2}\right)$ fixed, by Lemma 3.4 , one sees that problem $P_{\epsilon}\left(\tilde{\rho}, \tilde{\mu}_{\epsilon}, \tilde{d}_{\epsilon}\right)$ admits at least one solution $u_{\epsilon}$

Remark 3.5. Using the relation (3.12) and the fact that the functions $b$ and $\rho$ are non-decreasing, it follows that for $k$ large enough, the solution of the problem $P\left(\tilde{\rho}, \tilde{\mu}_{\epsilon}, \tilde{d}_{\epsilon}\right)$ belongs to $L^{\infty}(\Omega) \cap$ $L^{\infty}\left(\tilde{\Gamma}_{N e}\right)$ and $\left|u_{\epsilon}\right| \leq c\left(b, k_{1}\right)$ a.e. in $\Omega$ and $\left|u_{\epsilon}\right| \leq c\left(\rho, k_{2}\right)$ a.e. on $\tilde{\Gamma}_{N e}$.

Now, we set $\tilde{a}_{i}(x, \xi)=a_{i}(x, \xi) \chi_{\Omega}(x)+\frac{1}{\epsilon^{p_{i}(x)}}|\xi|^{p_{i}(x)-2} \xi \chi_{\tilde{\Omega} \backslash \Omega}(x)$ for all $(x, \xi) \in \tilde{\Omega} \times \mathbb{R}^{N}$ and we consider the following problem. $P_{\epsilon}\left(\tilde{\rho}, \tilde{\mu}_{\epsilon}, \tilde{d}_{\epsilon}\right)$

$$
\begin{cases}-\sum_{i=1}^{N} \frac{\partial}{\partial x_{i}}\left(a_{i}\left(x, \frac{\partial}{\partial x_{i}} u_{\epsilon}\right) \chi_{\Omega}(x)+\frac{1}{\epsilon p_{i}(x)}\left|\frac{\partial}{\partial x_{i}} u_{\epsilon}\right|^{p_{i}(x)-2} \frac{\partial}{\partial x_{i}} u_{\epsilon} \chi_{\tilde{\Omega} \backslash \Omega}(x)\right)+ & \\ \left|u_{\epsilon}\right|^{p_{M}(x)-2} u_{\epsilon} \chi_{\Omega}=\tilde{\mu}_{\epsilon} & \text { in } \tilde{\Omega} \\ u_{\epsilon}=0 & \text { on } \Gamma_{D} \\ \tilde{\rho}\left(u_{\epsilon}\right)+\sum_{i=1}^{N} \tilde{a}_{i}\left(x, \frac{\partial}{\partial x_{i}} u_{\epsilon}\right) \eta_{i}=\tilde{d}_{\epsilon} & \text { on } \tilde{\Gamma}_{N e} .\end{cases}
$$

Thanks to Theorem 3.2, $P_{\epsilon}\left(\tilde{\rho}, \tilde{\mu}_{\epsilon}, \tilde{d}_{\epsilon}\right)$ has at least one solution. So, there exists at least one measurable function $u_{\epsilon}: \tilde{\Omega} \rightarrow \mathbb{R}$ such that

$$
\left\{\begin{array}{l}
\sum_{i=1}^{N} \int_{\Omega} a_{i}\left(x, \frac{\partial}{\partial x_{i}} u_{\epsilon}\right) \frac{\partial}{\partial x_{i}} \tilde{\xi} d x+\sum_{i=1}^{N} \int_{\tilde{\Omega} \backslash \Omega}\left(\frac{1}{\epsilon^{p_{i}(x)}}\left|\frac{\partial}{\partial x_{i}} u_{\epsilon}\right|^{p_{i}(x)-2} \frac{\partial}{\partial x_{i}} u_{\epsilon} \cdot \frac{\partial}{\partial x_{i}} \tilde{\xi}\right) d x \\
+\int_{\Omega}\left|u_{\epsilon}\right|^{p_{M}(x)-2} u_{\epsilon} \tilde{\xi} d x=\int_{\Omega} \tilde{\xi} d \mu_{\epsilon}+\int_{\tilde{\Gamma}_{N e}}\left(\tilde{d}_{\epsilon}-\tilde{\rho}\left(u_{\epsilon}\right) \tilde{\xi} d \sigma\right.
\end{array}\right.
$$

where $u_{\epsilon} \in W_{D}^{1, \vec{p}(.)}(\tilde{\Omega})$ and $\tilde{\xi} \in W_{D}^{1, \vec{p}(.)}(\tilde{\Omega}) \cap L^{\infty}(\Omega)$.

Moreover $u_{\epsilon} \in L^{\infty}(\Omega) \cap L^{\infty}\left(\tilde{\Gamma}_{N e}\right)$.

Our aim is to prove that these approximated solutions $u_{\epsilon}$ tend, as $\epsilon$ goes to 0 , to a measurable function $u$ which is an entropy solution of the problem $P(\tilde{\rho}, \tilde{\mu}, \tilde{d})$. To start with, we establish some a priori estimates.

Proposition 3.6. Let $u_{\epsilon}$ be a solution of the problem $P_{\epsilon}\left(\tilde{\rho}, \tilde{\mu}_{\epsilon}, \tilde{d}_{\epsilon}\right)$. Then, the following statements hold.

(i) $\forall k>0$,

$$
\sum_{i=1}^{N} \int_{\Omega}\left|\frac{\partial}{\partial x_{i}} T_{k}\left(u_{\epsilon}\right)\right|^{p_{i}(x)} d x+\sum_{i=1}^{N} \int_{\tilde{\Omega} \backslash \Omega}\left(\frac{1}{\epsilon}\left|\frac{\partial}{\partial x_{i}} T_{k}\left(u_{\epsilon}\right)\right|\right)^{p_{i}(x)} d x \leq k\left(\|\tilde{d}\|_{L^{1}\left(\tilde{\Gamma}_{N e}\right)}+|\mu|(\Omega)\right) ;
$$


(ii)

$$
\int_{\Omega}\left|u_{\epsilon}\right|^{p_{M}(x)-1} d x+\int_{\tilde{\Gamma}_{N e}}\left|\tilde{\rho}\left(u_{\epsilon}\right)\right| d x \leq\left(\|\tilde{d}\|_{L^{1}\left(\tilde{\Gamma}_{N e}\right)}+|\mu|(\Omega)\right)
$$

(iii) $\forall k>0$,

$$
\sum_{i=1}^{N} \int_{\tilde{\Omega}}\left|\frac{\partial}{\partial x_{i}} T_{k}\left(u_{\epsilon}\right)\right|^{p_{i}(x)} d x \leq k\left(\|\tilde{d}\|_{L^{1}\left(\tilde{\Gamma}_{N e}\right)}+|\mu|(\Omega)\right) .
$$

Proof. For any $k>0$, we set $\tilde{\xi}=T_{k}\left(u_{\epsilon}\right)$ in (3.19), to get

$$
\left\{\begin{array}{l}
\sum_{i=1}^{N} \int_{\Omega}\left(a_{i}\left(x, \frac{\partial}{\partial x_{i}} u_{\epsilon}\right) \frac{\partial}{\partial x_{i}} T_{k}\left(u_{\epsilon}\right)\right) d x+\sum_{i=1}^{N} \int_{\tilde{\Omega} \backslash \Omega}\left(\frac{1}{\epsilon^{p_{i}(x)}}\left|\frac{\partial}{\partial x_{i}} u_{\epsilon}\right|^{p_{i}(x)-2} \frac{\partial}{\partial x_{i}} u_{\epsilon} \frac{\partial}{\partial x_{i}} T_{k}\left(u_{\epsilon}\right)\right) d x \\
\int_{\Omega}\left|u_{\epsilon}\right|^{p_{M}(x)-2} u_{\epsilon} T_{k}\left(u_{\epsilon}\right) d x=\int_{\Omega} T_{k}\left(u_{\epsilon}\right) d \mu_{\epsilon}+\int_{\tilde{\Gamma}_{N e}}\left(\tilde{d}_{\epsilon}-\tilde{\rho}\left(u_{\epsilon}\right)\right) T_{k}\left(u_{\epsilon}\right) d \sigma .
\end{array}\right.
$$

(i) Obviously, we have

$\sum_{i=1}^{N} \int_{\tilde{\Omega} \backslash \Omega}\left(\frac{1}{\epsilon^{p_{i}(x)}}\left|\frac{\partial}{\partial x_{i}} u_{\epsilon}\right|^{p_{i}(x)-2} \frac{\partial}{\partial x_{i}} u_{\epsilon} \frac{\partial}{\partial x_{i}} T_{k}\left(u_{\epsilon}\right)\right) d x=\sum_{i=1}^{N} \int_{\tilde{\Omega} \backslash \Omega}\left(\frac{1}{\epsilon^{p_{i}(x)}}\left|\frac{\partial}{\partial x_{i}} T_{k}\left(u_{\epsilon}\right)\right|^{p_{i}(x)}\right) d x \geq 0$,

$\int_{\tilde{\Gamma}_{N e}} \tilde{\rho}\left(u_{\epsilon}\right) T_{k}\left(u_{\epsilon}\right) d \sigma \geq 0$ and $\int_{\Omega}\left|u_{\epsilon}\right|^{p_{M}(x)-2} u_{\epsilon} T_{k}\left(u_{\epsilon}\right) d x \geq 0$.

Moreover,

$$
\left\{\begin{aligned}
\int_{\Omega} T_{k}\left(u_{\epsilon}\right) d \mu_{\epsilon}+\int_{\tilde{\Gamma}_{N e}} \tilde{d}_{\epsilon} T_{k}\left(u_{\epsilon}\right) d \sigma & \leq k \int_{\Omega} d \mu_{\epsilon}+k \int_{\tilde{\Gamma}_{N e}}\left|\tilde{d}_{\epsilon}\right| d \sigma \\
& \leq k\left(|\mu|(\Omega)+\int_{\tilde{\Gamma}_{N e}}|\tilde{d}| d \sigma\right) .
\end{aligned}\right.
$$

Using the inequalities above and (1.7), it follows that

$$
\sum_{i=1}^{N} \int_{\Omega}\left|\frac{\partial T_{k}\left(u_{\epsilon}\right)}{\partial x_{i}}\right|^{p_{i}(x)} d x \leq k\left(|\mu|(\Omega)+\int_{\tilde{\Gamma}_{N e}}|\tilde{d}| d \sigma\right) .
$$

As $\sum_{i=1}^{N} \int_{\Omega}\left(a_{i}\left(x, \frac{\partial}{\partial x_{i}} u_{\epsilon}\right) \frac{\partial}{\partial x_{i}} T_{k}\left(u_{\epsilon}\right)\right) d x \geq 0, \int_{\tilde{\Gamma}_{N e}} \tilde{\rho}\left(u_{\epsilon}\right) T_{k}\left(u_{\epsilon}\right) d \sigma \geq 0$ and $\int_{\Omega}\left|u_{\epsilon}\right|^{p_{M}(x)-2} u_{\epsilon} T_{k}\left(u_{\epsilon}\right) d x \geq 0$, therefore, we get from (3.20),

$$
\sum_{i=1}^{N} \int_{\tilde{\Omega} \backslash \Omega}\left(\frac{1}{\epsilon^{p_{i}(x)}}\left|\frac{\partial}{\partial x_{i}} T_{k}\left(u_{\epsilon}\right)\right|^{p_{i}(x)}\right) d x \leq k\left(|\mu|(\Omega)+\int_{\tilde{\Gamma}_{N e}}|\tilde{d}| d \sigma\right)
$$

Adding (3.22) and (3.23), we obtain (i).

(ii) The first two terms in (3.20) are non-negative and using (3.21), we have from (3.20) the following

$$
\int_{\tilde{\Gamma}_{N e}} \tilde{\rho}\left(u_{\epsilon}\right) T_{k}\left(u_{\epsilon}\right) d \sigma+\int_{\Omega}\left|u_{\epsilon}\right|^{p_{M}(x)-2} u_{\epsilon} T_{k}\left(u_{\epsilon}\right) d x \leq k\left(|\mu|(\Omega)+\int_{\tilde{\Gamma}_{N e}}|\tilde{d}| d \sigma\right) .
$$

We divide the above inequality by $k>0$ and let $k$ go to zero, to get

$$
\begin{aligned}
\int_{\tilde{\Gamma}_{N e}} \tilde{\rho}\left(u_{\epsilon}\right) \operatorname{sign}\left(u_{\epsilon}\right) d \sigma+\int_{\Omega}\left|u_{\epsilon}\right|^{p_{M}(x)-2} u_{\epsilon} \operatorname{sign}\left(u_{\epsilon}\right) d x & =\int_{\tilde{\Gamma}_{N e}}\left|\tilde{\rho}\left(u_{\epsilon}\right)\right| d \sigma+\int_{\Omega}\left|u_{\epsilon}\right|^{p_{M}(x)-1} d x \\
& \leq\left(|\mu|(\Omega)+\int_{\tilde{\Gamma}_{N e}}|\tilde{d}| d \sigma\right) .
\end{aligned}
$$


(iii) For all $k>0$, we have

$$
\sum_{i=1}^{N} \int_{\tilde{\Omega}}\left|\frac{\partial}{\partial x_{i}} T_{k}\left(u_{\epsilon}\right)\right|^{p_{i}(x)} d x \leq \sum_{i=1}^{N} \int_{\Omega}\left|\frac{\partial}{\partial x_{i}} T_{k}\left(u_{\epsilon}\right)\right|^{p_{i}(x)} d x+\sum_{i=1}^{N} \int_{\tilde{\Omega} \backslash \Omega}\left|\frac{1}{\epsilon} \frac{\partial}{\partial x_{i}} T_{k}\left(u_{\epsilon}\right)\right|^{p_{i}(x)} d x,
$$

for any $0<\epsilon<1$. According to $(i)$, we deduce that

$$
\sum_{i=1}^{N} \int_{\tilde{\Omega}}\left|\frac{\partial}{\partial x_{i}} T_{k}\left(u_{\epsilon}\right)\right|^{p_{i}(x)} d x \leq k\left(|\mu|(\Omega)+\int_{\tilde{\Gamma}_{N e}}|\tilde{d}| d \sigma\right) .
$$

Lemma 3.7. There is a positive constant D such that

$$
\operatorname{meas}\left\{\left|u_{\epsilon}\right|>k\right\} \leq D^{p_{m}^{-}} \frac{(1+k)}{k^{p_{m}^{-}-1}}, \forall k>0 .
$$

Proof. Let $k>0$; by using Proposition 3.6-(iii), we have

$$
\begin{aligned}
\sum_{i=1}^{N} \int_{\tilde{\Omega}}\left|\frac{\partial T_{k}\left(u_{\epsilon}\right)}{\partial x_{i}}\right|^{p_{m}^{-}(x)} d x & \leq \sum_{i=1}^{N}\left\{\left|\frac{\partial T_{k}\left(u_{\epsilon}\right)}{\partial x_{i}}\right|_{>1}\right\}\left|\frac{\partial T_{k}\left(u_{\epsilon}\right)}{\partial x_{i}}\right|^{p_{m}^{-}(x)} d x+\operatorname{Nmeas}(\tilde{\Omega}) \\
& \leq \sum_{i=1}^{N} \int_{\tilde{\Omega}}\left|\frac{\partial T_{k}\left(u_{\epsilon}\right)}{\partial x_{i}}\right|^{p_{i}(x)} d x+\operatorname{Nmeas}(\tilde{\Omega}) \\
& \leq k\left(|\mu|(\Omega)+\int_{\tilde{\Gamma}_{N e}}|\tilde{d}| d \sigma\right)+\operatorname{Nmeas}(\tilde{\Omega}) \\
& \leq C^{\prime}(k+1),
\end{aligned}
$$

with $C^{\prime}=\max \left(\left(|\mu|(\Omega)+\int_{\tilde{\Gamma}_{N e}}|\tilde{d}| d \sigma\right) ; N \operatorname{meas}(\tilde{\Omega})\right)$.

We can write the above inequality as

$$
\sum_{i=1}^{N}\left\|\frac{\partial T_{k}\left(u_{\epsilon}\right)}{\partial x_{i}}\right\|_{p_{m}^{-}}^{p_{m}^{-}} \leq C^{\prime}(1+k) \text { or }\left\|T_{k}\left(u_{\epsilon}\right)\right\|_{W_{D}^{1, p_{\bar{m}}^{-}}(\tilde{\Omega})} \leq\left[C^{\prime}(1+k)\right]^{\frac{1}{p^{-}}}
$$

By the Poincaré inequality in constant exponent, we obtain

$$
\left\|T_{k}\left(u_{\epsilon}\right)\right\|_{L^{p_{m}^{-}(\tilde{\Omega})}} \leq D(1+k)^{\frac{1}{p_{m}^{-}}} .
$$

The above inequality implies that

$$
\int_{\tilde{\Omega}}\left|T_{k}\left(u_{\epsilon}\right)\right|^{p_{m}^{-}} d x \leq D^{p_{m}^{-}}(1+k),
$$

from which we obtain

$$
\text { meas }\left\{\left|u_{\epsilon}\right|>k\right\} \leq D^{p_{m}^{-}} \frac{(1+k)}{k^{p_{m}^{-}}},
$$

since

$$
\int_{\tilde{\Omega}}\left|T_{k}\left(u_{\epsilon}\right)\right|^{p_{m}^{-}} d x=\int_{\left\{\left|u_{\epsilon}\right|>k\right\}}\left|T_{k}\left(u_{\epsilon}\right)\right|^{p_{m}^{-}} d x+\int_{\left\{\left|u_{\epsilon}\right| \leq k\right\}}\left|T_{k}\left(u_{\epsilon}\right)\right|^{p_{m}^{-}} d x,
$$


we get

$$
\int_{\left\{\left|u_{\epsilon}\right|>k\right\}}\left|T_{k}\left(u_{\epsilon}\right)\right|^{p_{m}^{-}} d x \leq \int_{\tilde{\Omega}}\left|T_{k}\left(u_{\epsilon}\right)\right|^{p_{m}^{-}} d x
$$

and

$$
k^{p_{m}^{-}} \text {meas }\left\{\left|u_{\epsilon}\right|>k\right\} \leq \int_{\tilde{\Omega}}\left|T_{k}\left(u_{\epsilon}\right)\right|^{p_{m}^{-}} d x \leq D^{p_{m}^{-}}(1+k)
$$

Lemma 3.8. There is a positive constant $C$ such that

$$
\sum_{i=1}^{N} \int_{\tilde{\Omega}}\left(\left|\frac{\partial}{\partial x_{i}} T_{k}\left(u_{\epsilon}\right)\right|^{p_{i}^{-}}\right) d x \leq C(k+1), \forall k>0 .
$$

Proof. Let $k>0$, we set $\Omega_{1}=\left\{|u| \leq k ;\left|\frac{\partial}{\partial x_{i}} u_{\epsilon}\right| \leq 1\right\}$ and $\Omega_{2}=\left\{|u| \leq k ;\left|\frac{\partial}{\partial x_{i}} u_{\epsilon}\right|>1\right\}$; using Proposition 3.6-(iii), we have

$$
\begin{aligned}
\sum_{i=1}^{N} \int_{\tilde{\Omega}}\left(\left|\frac{\partial}{\partial x_{i}} T_{k}\left(u_{\epsilon}\right)\right|^{p_{i}^{-}}\right) d x & =\sum_{i=1}^{N} \int_{\Omega_{1}}\left(\left|\frac{\partial}{\partial x_{i}} T_{k}\left(u_{\epsilon}\right)\right|^{p_{i}^{-}}\right) d x+\sum_{i=1}^{N} \int_{\Omega_{2}}\left(\left|\frac{\partial}{\partial x_{i}} T_{k}\left(u_{\epsilon}\right)\right|^{p_{i}^{-}}\right) d x \\
& \leq \operatorname{Nmeas}(\tilde{\Omega})+\sum_{i=1}^{N} \int_{\tilde{\Omega}}\left(\left|\frac{\partial}{\partial x_{i}} T_{k}\left(u_{\epsilon}\right)\right|^{p_{i}(x)}\right) d x \\
& \leq \operatorname{Nmeas}(\tilde{\Omega})+k\left(|\mu|(\Omega)+\|\tilde{d}\|_{L^{1}\left(\tilde{\Gamma}_{N e}\right)}\right) \leq C(k+1),
\end{aligned}
$$

with $C=\max \left\{\operatorname{Nmeas}(\tilde{\Omega}) ;\left(|\mu|(\Omega)+\|\tilde{d}\|_{L^{1}\left(\tilde{\Gamma}_{N e}\right)}\right)\right\}$.

Lemma 3.9. For all $k>0$, there is two constants $C_{1}$ and $C_{2}$ such that

(i) $\left\|u_{\epsilon}\right\|_{\mathcal{M}^{q^{*}}(\tilde{\Omega})} \leq C_{1}$;

(ii) $\left\|\frac{\partial}{\partial x_{i}} u_{\epsilon}\right\|_{\mathcal{M}^{p_{i}^{-} q / p}(\tilde{\Omega})} \leq C_{2}$.

Proof. (i) By Lemma 3.8, we have

$$
\sum_{i=1}^{N} \int_{\tilde{\Omega}}\left|\frac{\partial}{\partial x_{i}} T_{k}\left(u_{\epsilon}\right)\right|^{p_{i}^{-}} d x \leq C(1+k), \forall k>0 \text { and } i=1, \ldots, N
$$

- If $k>1$, we have

$$
\sum_{i=1}^{N} \int_{\tilde{\Omega}}\left|\frac{\partial}{\partial x_{i}} T_{k}\left(u_{\epsilon}\right)\right|^{p_{i}^{-}} d x \leq C^{\prime} k,
$$

which means $T_{k}\left(u_{\epsilon}\right) \in W^{1,\left(p_{1}^{-}, \ldots, p_{N}^{-}\right)}(\tilde{\Omega})$. Using relation (2.8), we deduce that

$$
\left\|T_{k}\left(u_{\epsilon}\right)\right\|_{L^{(\bar{p})^{*}(\tilde{\Omega}}} \leq C_{1} \prod_{i=1}^{N}\left\|\frac{\partial}{\partial x_{i}} T_{k}\left(u_{\epsilon}\right)\right\|_{L^{p_{i}^{-}(\tilde{\Omega})}}^{\frac{1}{N}} .
$$


So,

$$
\begin{aligned}
\int_{\tilde{\Omega}}\left|T_{k}\left(u_{\epsilon}\right)\right|^{(\bar{p})^{*}} d x & \leq C\left[\prod_{i=1}^{N}\left(\int_{\tilde{\Omega}}\left|\frac{\partial}{\partial x_{i}} T_{k}\left(u_{\epsilon}\right)\right|^{p_{i}^{-}} d x\right)^{\frac{1}{N p_{i}^{-}}}\right]^{(\bar{p})^{*}} \\
& \leq C^{\prime \prime}\left[\prod_{i=1}^{N}(k) \frac{1}{N p_{i}^{-}}\right]^{(\bar{p})^{*}} \\
& \leq C^{\prime \prime}\left[\sum_{k^{i=1}}^{N} \frac{1}{N p_{i}^{-}}\right]^{(\bar{p})^{*}} \\
& \leq C^{\prime \prime} k \frac{(\bar{p})^{*}}{\bar{p}} .
\end{aligned}
$$

Thus,

$$
\begin{aligned}
\int_{\left\{\left|u_{\epsilon}\right|>k\right\}}\left|T_{k}\left(u_{\epsilon}\right)\right|^{(\bar{p})^{*}} d x & \leq \int_{\tilde{\Omega}}\left|T_{k}\left(u_{\epsilon}\right)\right|^{(\bar{p})^{*}} d x \\
& \leq C^{\prime} k^{\frac{(\bar{p})^{*}}{\bar{p}}}
\end{aligned}
$$

and so,

$$
(k)^{(\bar{p})^{*}} \operatorname{meas}\left\{x \in \tilde{\Omega}:\left|u_{\epsilon}\right|>k\right\} \leq C^{\prime} k^{\frac{(\bar{p})^{*}}{\bar{p}}}
$$

which means that

$$
\lambda_{u_{\epsilon}}(k) \leq C^{\prime} k^{(\bar{p})^{*}\left(\frac{1}{\bar{p}}-1\right)}=C^{\prime} k^{-q^{*}}, \forall k \geq 1
$$

- If $0<k<1$, we have

$$
\begin{aligned}
\lambda_{u_{\epsilon}}(k) & =\operatorname{meas}\left\{x \in \tilde{\Omega}:\left|u_{\epsilon}\right|>k\right\} \\
& \leq \operatorname{meas}(\tilde{\Omega}) \\
& \leq \operatorname{meas}(\tilde{\Omega}) k^{-q^{*}} .
\end{aligned}
$$

So,

$$
\lambda_{u_{\epsilon}}(k) \leq\left(C^{\prime}+\operatorname{meas}(\tilde{\Omega})\right) k^{-q^{*}}=C_{1} k^{-q^{*}} .
$$

Therefore,

$$
\left\|u_{\epsilon}\right\|_{\mathcal{M}^{q^{*}(\tilde{\Omega})}} \leq C_{1}
$$


(ii) $\bullet$ Let $\alpha \geq 1$. For all $k \geq 1$, we have

$$
\begin{aligned}
\lambda_{\frac{\partial u_{\epsilon}}{\partial x_{i}}}(\alpha) & =\operatorname{meas}\left(\left\{\left|\frac{\partial u_{\epsilon}}{\partial x_{i}}\right|>\alpha\right\}\right) \\
& =\operatorname{meas}\left(\left\{\left|\frac{\partial u_{\epsilon}}{\partial x_{i}}\right|>\alpha ;\left|u_{\epsilon}\right| \leq k\right\}\right)+\operatorname{meas}\left(\left\{\left|\frac{\partial u_{\epsilon}}{\partial x_{i}}\right|>\alpha ;\left|u_{\epsilon}\right|>k\right\}\right) \\
& \leq \int\left\{\left|\frac{\partial u_{\epsilon}}{\partial x_{i}}\right|>\alpha ;\left|u_{\epsilon}\right| \leq k\right\} \\
& \leq \int_{\left\{\left|u_{\epsilon}\right| \leq k\right\}}\left(\frac{1}{\alpha}\left|\frac{\partial u_{\epsilon}}{\partial x_{i}}\right|\right)^{p_{i}^{-}} d x+\lambda_{u_{\epsilon}}(k) \\
& \leq \alpha^{-p_{i}^{-}} C^{\prime} k+C k^{-q^{*}} \\
& \leq B\left(\alpha^{-p_{i}^{-}} k+k^{-q^{*}}\right),
\end{aligned}
$$

with $B=\max \left(C^{\prime} ; C\right)$.

Let $g:[1 ; \infty) \rightarrow \mathbb{R}, x \mapsto g(x)=\frac{x}{\alpha^{p_{i}^{-}}}+x^{-q^{*}}$.

We have $g^{\prime}(x)=0$ with $x=\left(q^{*} \alpha^{p^{-}}\right) \frac{1}{q^{*}+1}$.

We set $k=\left(q^{*} \alpha^{p_{i}^{-}}\right)^{\frac{1}{q^{*}+1}} \geq 1$ in the above inequality to get,

$$
\begin{aligned}
\lambda_{\frac{\partial u_{\epsilon}}{\partial x_{i}}}(\alpha) & \leq B\left[\alpha^{-p_{i}^{-}} \times\left(q^{*} \alpha^{p_{i}^{-}}\right) \frac{1}{q^{*}+1}+\left(q^{*} \alpha^{p_{i}^{-}}\right) \frac{-q^{*}}{q^{*}+1}\right] \\
& \leq B\left[\left(q^{*}\right)^{\frac{1}{q^{*}+1}} \times \alpha^{\left.-p_{i}^{-}\left(1-\frac{1}{q^{*}+1}\right)+\left(q^{*}\right) \frac{-q^{*}}{q^{*}+1} \times \alpha \frac{-p_{i}^{-} q^{*}}{q^{*}+1}\right]}\right. \\
& \leq B\left[\left(q^{*}\right)^{\frac{1}{q^{*}+1}} \times \alpha^{-p_{i}^{-}\left(\frac{q^{*}}{q^{*}+1}\right)}+\left(q^{*} \frac{-q^{*}}{q^{*}+1} \times \alpha \frac{-p_{i}^{-} q^{*}}{q^{*}+1}\right]\right. \\
& \leq M \alpha^{-p_{i}^{-}} \frac{q^{*}}{q^{*}+1} \\
& \leq M \alpha^{-p_{i}^{-}} \frac{q}{\bar{p}}
\end{aligned}
$$

where $M=B \times \max \left(\left(q^{*}\right)^{\frac{1}{q^{*}+1}} ;\left(q^{*}\right)^{\frac{-q^{*}}{q^{*}+1}}\right)$ and as $q^{*}=\frac{N(\bar{p}-1)}{N-\bar{p}}, q=\frac{N(\bar{p}-1)}{N-1}$. 
So,

$$
\begin{aligned}
\frac{q^{*}}{q^{*}+1} & =\frac{q^{*}(N-\bar{p})}{N(\bar{p}-1)+N-\bar{p}} \\
& =\frac{q^{*}(N-\bar{p})}{N \bar{p}-\bar{p}} \\
& =\frac{N(\bar{p}-1)}{(N-1) \bar{p}} \\
& =\frac{q}{\bar{p}} .
\end{aligned}
$$

- If $0 \leq \alpha<1$, we have.

$$
\begin{aligned}
\lambda_{\frac{\partial u_{\epsilon}}{\partial x_{i}}}(\alpha) & =\operatorname{meas}\left(\left\{x \in \tilde{\Omega}:\left|\frac{\partial u_{\epsilon}}{\partial x_{i}}\right|>\alpha\right\}\right) \\
& \leq \operatorname{meas}(\tilde{\Omega}) \alpha^{-p_{i}^{-}} \frac{q}{\bar{p}}
\end{aligned}
$$

Therefore,

$$
\lambda_{\frac{\partial u_{\epsilon}}{\partial x_{i}}}(\alpha) \leq(M+\operatorname{meas}(\tilde{\Omega})) \alpha^{-p_{i}^{-}} \frac{q}{\bar{p}}, \forall \alpha \geq 0
$$

So,

$$
\left\|\frac{\partial u_{\epsilon}}{\partial x_{i}}\right\|_{H} \leq C_{2}
$$

where $H=\mathcal{M}(\tilde{\Omega}) \frac{p_{i}^{-} q}{\bar{p}}$

Proposition 3.10. Let $u_{\epsilon}$ be a solution of the problem $P\left(\tilde{\rho}, \tilde{\mu}_{\epsilon}, \tilde{d}_{\epsilon}\right)$. Then,

(i) $u_{\epsilon} \rightarrow u$ in measure, a.e. in $\Omega$ and a.e. on $\tilde{\Gamma}_{N}$;

(ii) For all $i=1, \ldots N, \frac{\partial T_{k}\left(u_{\epsilon}\right)}{\partial x_{i}} \rightarrow \frac{\partial T_{k}(u)}{\partial x_{i}}=0$ in $L^{p_{i}^{-}}(\tilde{\Omega} \backslash \Omega)$.

Proof. (i) By Proposition $3.6(i)$, we deduce that $\left(T_{k}\left(u_{\epsilon}\right)\right)_{\epsilon>0}$ is bounded in $W_{D}^{1, \vec{p}(.)}(\tilde{\Omega}) \hookrightarrow$ $L^{p_{m}(.)}(\tilde{\Omega}) \hookrightarrow L^{p_{m}^{-}}(\tilde{\Omega})$ (with compact embedding). Therefore, up to a subsequence, we can assume that as $\epsilon \rightarrow 0,\left(T_{k}\left(u_{\epsilon}\right)\right)_{\epsilon>0}$ converges strongly to some function $\sigma_{k}$ in $L^{p_{m}^{-}}(\tilde{\Omega})$, a.e. in $\tilde{\Omega}$ and a.e. on $\tilde{\Gamma}_{N e}$.

Let us see that the sequence $\left(u_{\epsilon}\right)_{\epsilon>0}$ is Cauchy in measure.

Indeed, let $s>0$ and define:

$E_{1}=\left[\left|u_{\epsilon_{1}}\right|>k\right], E_{2}=\left[\left|u_{\epsilon_{2}}\right|>k\right]$ and $E_{3}=\left[\left|T_{k}\left(u_{\epsilon_{1}}\right)-T_{k}\left(u_{\epsilon_{2}}\right)\right|>s\right]$,

where $k>0$ is fixed. We note that

$$
\left[\left|u_{\epsilon_{1}}-u_{\epsilon_{2}}\right|>s\right] \subset E_{1} \cup E_{2} \cup E_{3} ;
$$


hence,

$$
\operatorname{meas}\left(\left[\left|u_{\epsilon_{1}}-u_{\epsilon_{2}}\right|>s\right]\right) \leq \sum_{i=1}^{3} \operatorname{meas}\left(E_{i}\right) .
$$

Let $\theta>0$, using Lemma 3.7, we choose $k=k(\theta)$ such that

$$
\operatorname{meas}\left(E_{1}\right) \leq \frac{\theta}{3} \text { and meas }\left(E_{2}\right) \leq \frac{\theta}{3}
$$

Since $\left(T_{k}\left(u_{\epsilon}\right)\right)_{\epsilon>0}$ converges strongly in $L^{p_{m}^{-}}(\tilde{\Omega})$, then, it is a Cauchy sequence in $L^{p_{m}^{-}}(\tilde{\Omega})$. Thus,

$$
\operatorname{meas}\left(E_{3}\right) \leq \frac{1}{s^{p_{m}^{-}}} \int_{\Omega}\left|T_{k}\left(u_{\epsilon_{1}}\right)-T_{k}\left(u_{\epsilon_{2}}\right)\right|^{p_{m}^{-}} d x \leq \frac{\theta}{3},
$$

for all $\epsilon_{1}, \epsilon_{2} \geq n_{0}(s, \theta)$. Finally, from $(3.25)$, (3.26) and (3.27), we obtain

$$
\operatorname{meas}\left(\left[\left|u_{\epsilon_{1}}-u_{\epsilon_{2}}\right|>s\right]\right) \leq \theta \text { for all } \epsilon_{1}, \epsilon_{2} \geq n_{0}(s, \theta)
$$

which means that the sequence $\left(u_{\epsilon}\right)_{\epsilon>0}$ is Cauchy in measure, so $u_{\epsilon} \rightarrow u$ in measure and up to a subsequence, we have $u_{\epsilon} \rightarrow u$ a.e. in $\tilde{\Omega}$. Hence, $\sigma_{k}=T_{k}(u)$ a.e. in $\tilde{\Omega}$ and so, $u \in \mathcal{T}_{D}^{1, \vec{p}(.)}(\Omega)$.

(ii) According to the proof of (i), we have $T_{k}\left(u_{\epsilon}\right) \rightarrow T_{k}(u)$ in $W_{D}^{1, \vec{p}(.)}(\tilde{\Omega}) \hookrightarrow W_{D}^{1, \vec{p}_{-}}(\tilde{\Omega})$ which implies on one hand that for all $i=1, \ldots N, \frac{\partial T_{k}\left(u_{\epsilon}\right)}{\partial x_{i}} \rightarrow \frac{\partial T_{k}(u)}{\partial x_{i}}$ in $L^{p_{i}(.)}(\tilde{\Omega})$ and on the other hand that for all $i=1, \ldots N, \frac{\partial T_{k}\left(u_{\epsilon}\right)}{\partial x_{i}} \rightarrow \frac{\partial T_{k}(u)}{\partial x_{i}}$ in $L^{p_{i}(.)}(\tilde{\Omega})$ and then for all $i=1, \ldots N$, $\frac{\partial T_{k}\left(u_{\epsilon}\right)}{\partial x_{i}} \rightarrow \frac{\partial T_{k}(u)}{\partial x_{i}}$ in $L^{p_{i}^{-}}(\tilde{\Omega} \backslash \Omega)$.

Let $i=1, \ldots, N$, by Proposition 3.6-(i), we can assert that $\left(\frac{1}{\epsilon} \frac{\partial T_{k}\left(u_{\epsilon}\right)}{\partial x_{i}}\right)_{\epsilon>0}$ is bounded in $L^{p_{i}^{-}}(\tilde{\Omega} \backslash \Omega)$. Indeed, let $k>0$, we set $\Omega^{1}=\left\{x \in \tilde{\Omega} \backslash \Omega ;|u(x)| \leq k ;\left|\frac{\partial}{\partial x_{i}} u_{\epsilon}(x)\right| \leq \epsilon\right\}$ and $\Omega^{2}=\left\{x \in \tilde{\Omega} \backslash \Omega ;|u| \leq k ;\left|\frac{\partial}{\partial x_{i}} u_{\epsilon}(x)\right|>\epsilon\right\} ;$ using Proposition 3.6-(i), we have

$$
\begin{aligned}
& \sum_{i=1}^{N} \int_{\tilde{\Omega} \backslash \Omega}\left(\frac{1}{\epsilon}\left|\frac{\partial T_{k}\left(u_{\epsilon}\right)}{\partial x_{i}}\right|^{p_{i}^{-}}\right) d x \\
= & \sum_{i=1}^{N} \int_{\Omega^{1}}\left(\frac{1}{\epsilon}\left|\frac{\partial T_{k}\left(u_{\epsilon}\right)}{\partial x_{i}}\right|^{p_{i}^{-}}\right) d x+\sum_{i=1}^{N} \int_{\Omega^{2}}\left(\frac{1}{\epsilon}\left|\frac{\partial}{\partial x_{i}} T_{k}\left(u_{\epsilon}\right)\right|^{p_{i}^{-}}\right) d x \\
\leq & N \operatorname{meas}(\tilde{\Omega} \backslash \Omega)+\sum_{i=1}^{N} \int_{\tilde{\Omega} \backslash \Omega}\left(\frac{1}{\epsilon}\left|\frac{\partial}{\partial x_{i}} T_{k}\left(u_{\epsilon}\right)\right|^{p_{i}(x)}\right) d x \\
\leq & N \operatorname{meas}(\tilde{\Omega} \backslash \Omega)+k\left(|\mu|(\Omega)+\|\tilde{d}\|_{L^{1}\left(\tilde{\Gamma}_{N e}\right)}\right) \leq C^{\prime}(k+1),
\end{aligned}
$$

with $C^{\prime}=\max \left\{N \operatorname{meas}(\tilde{\Omega} \backslash \Omega) ;\left(|\mu|(\Omega)+\|\tilde{d}\|_{L^{1}\left(\tilde{\Gamma}_{N e}\right)}\right)\right\}$. To end, we have

$$
\int_{\tilde{\Omega} \backslash \Omega}\left(\frac{1}{\epsilon}\left|\frac{\partial T_{k}\left(u_{\epsilon}\right)}{\partial x_{i}}\right|^{p_{i}^{-}}\right) d x \leq \sum_{i=1}^{N} \int_{\tilde{\Omega} \backslash \Omega}\left(\frac{1}{\epsilon}\left|\frac{\partial T_{k}\left(u_{\epsilon}\right)}{\partial x_{i}}\right|^{p_{i}^{-}}\right) d x, \text { for any } i=1, \ldots, N
$$


Therefore, there exists $\Theta_{k} \in L^{p_{i}^{-}}(\tilde{\Omega} \backslash \Omega)$ such that

$$
\frac{1}{\epsilon} \frac{\partial T_{k}\left(u_{\epsilon}\right)}{\partial x_{i}} \rightarrow \Theta_{k} \text { in } L^{p_{i}^{-}}(\tilde{\Omega} \backslash \Omega) \text { as } \epsilon \rightarrow 0 .
$$

For any $\psi \in L^{\left(p_{i}^{\prime}\right)^{-}}(\tilde{\Omega} \backslash \Omega)$, we have

$$
\int_{\tilde{\Omega} \backslash \Omega} \frac{\partial T_{k}\left(u_{\epsilon}\right)}{\partial x_{i}} \psi d x=\int_{\tilde{\Omega} \backslash \Omega}\left(\frac{1}{\epsilon} \frac{\partial T_{k}\left(u_{\epsilon}\right)}{\partial x_{i}}-\Theta_{k}\right)(\epsilon \psi) d x+\epsilon \int_{\tilde{\Omega} \backslash \Omega} \Theta_{k} \psi d x .
$$

As $(\epsilon \psi)_{\epsilon>0}$ converges strongly to zero in $L^{\left(p_{i}^{\prime}\right)^{-}}(\tilde{\Omega} \backslash \Omega)$, we pass to the limit as $\epsilon \rightarrow 0$ in (3.29), to get

$$
\frac{\partial T_{k}\left(u_{\epsilon}\right)}{\partial x_{i}} \rightarrow 0 \text { in } L^{p_{i}^{-}}(\tilde{\Omega} \backslash \Omega) .
$$

Hence, one has

$$
\frac{\partial T_{k}\left(u_{\epsilon}\right)}{\partial x_{i}} \rightarrow \frac{\partial T_{k}(u)}{\partial x_{i}}=0 \text { in } L^{p_{i}^{-}}(\tilde{\Omega} \backslash \Omega),
$$

for any $i=1, \ldots, N$.

Lemma 3.11. $b(u) \in L^{1}(\Omega)$ and $\tilde{\rho}(u) \in L^{1}\left(\tilde{\Gamma}_{N e}\right)$.

Proof. Having in mind that by Proposition 3.6-(ii),

$$
\int_{\Omega}\left|b\left(u_{\epsilon}\right)\right| d x+\int_{\tilde{\Gamma}_{N e}}\left|\tilde{\rho}\left(u_{\epsilon}\right)\right| d \sigma \leq\left(|\mu|(\Omega)+\|\tilde{d}\|_{L^{1}\left(\tilde{\Gamma}_{N e}\right)}\right),
$$

we deduce that

$$
\int_{\Omega}\left|b\left(u_{\epsilon}\right)\right| d x \leq\left(|\mu|(\Omega)+\|\tilde{d}\|_{L^{1}\left(\tilde{\Gamma}_{N e}\right)}\right)
$$

and

$$
\int_{\tilde{\Gamma}_{N e}}\left|\tilde{\rho}\left(u_{\epsilon}\right)\right| d \sigma \leq\left(|\mu|(\Omega)+\|\tilde{d}\|_{L^{1}\left(\tilde{\Gamma}_{N e}\right)}\right) .
$$

By Fatou's lemma, the continuity of $b, \tilde{\rho}$ and using Proposition 3.10, we have

$$
\liminf _{\epsilon \rightarrow 0} \int_{\Omega}\left|b\left(u_{\epsilon}\right)\right| d x \geq \int_{\Omega}|b(u)| d x
$$

and

$$
\liminf _{\epsilon \rightarrow 0} \int_{\tilde{\Gamma}_{N e}}\left|\tilde{\rho}\left(u_{\epsilon}\right)\right| d \sigma \geq \int_{\tilde{\Gamma}_{N e}}|\tilde{\rho}(u)| d \sigma .
$$

Using (3.30)-(3.33), we deduce that

$$
\int_{\Omega}|b(u)| d x \leq\left(|\mu|(\Omega)+\|\tilde{d}\|_{L^{1}\left(\tilde{\Gamma}_{N e}\right)}\right)
$$

and

$$
\int_{\tilde{\Gamma}_{N e}}|\tilde{\rho}(u)| d \sigma \leq\left(|\mu|(\Omega)+\|\tilde{d}\|_{L^{1}\left(\tilde{\Gamma}_{N e}\right)}\right) .
$$

Therefore, $b(u) \in L^{1}(\Omega)$ and $\tilde{\rho}(u) \in L^{1}\left(\tilde{\Gamma}_{N e}\right)$. 
Lemma 3.12. Assume (1.4)-(1.8) hold and $u_{\epsilon}$ be a weak solution of the problem $P\left(\rho, \tilde{\mu}_{\epsilon}, \tilde{d}_{\epsilon}\right)$. Then,

(i) $\frac{\partial}{\partial x_{i}} u_{\epsilon}$ converges in measure to $\frac{\partial}{\partial x_{i}} u$.

(ii) $a_{i}\left(x, \frac{\partial T_{k}\left(u_{\epsilon}\right)}{\partial x_{i}}\right) \rightarrow a_{i}\left(x, \frac{\partial T_{k}(u)}{\partial x_{i}}\right)$ strongly in $L^{1}(\Omega)$ and weakly in $L^{p_{i}^{\prime}(.)}(\Omega)$, for all $i=1, \ldots, N$.

In order to give the proof of Lemma 3.12, we need the following lemmas.

Lemma 3.13 (Cf $[6])$. Let $u \in \mathcal{T}^{1, \vec{p}(.)}(\Omega)$. Then, there exists a unique measurable function $\nu_{i}: \Omega \rightarrow \mathbb{R}$ such that

$$
\nu_{i} \chi_{\{|u|<k\}}=\frac{\partial}{\partial x_{i}} T_{k}(u) \text { for a.e. } x \in \Omega, \forall k>0 \text { and } i=1, \ldots, N \text {; }
$$

where $\chi_{A}$ denotes the characteristic function of a measurable set $A$.

The functions $\nu_{i}$ are denoted $\frac{\partial}{\partial x_{i}} u$. Moreover, if u belongs to $W^{1, \vec{p}(.)}(\Omega)$, then $\nu_{i} \in L^{p_{i}(.)}(\Omega)$ and coincides with the standard distributional gradient of $u$ i.e. $\nu_{i}=\frac{\partial}{\partial x_{i}} u$.

Lemma 3.14 (Cf [37], lemma 5.4). Let $\left(v_{n}\right)_{n \in \mathbb{N}}$ be a sequence of measurable functions. If $v_{n}$ converges in measure to $v$ and is uniformly bounded in $L^{p(.)}(\Omega)$ for some $1<<p(.) \in L^{\infty}(\Omega)$, then $v_{n} \rightarrow v$ strongly in $L^{1}(\Omega)$.

The third technical lemma is a standard fact in measure theory ( $\mathrm{Cf}[16])$.

Lemma 3.15. Let $(X, \mathcal{M}, \mu)$ be a measurable space such that $\mu(X)<\infty$.

Consider a measurable function $\gamma: X \rightarrow[0 ; \infty]$ such that

$$
\mu(\{x \in X: \gamma(x)=0\})=0 .
$$

Then, for every $\epsilon>0$, there exists $\delta$ such that

$$
\mu(A)<\epsilon, \text { for all } A \in \mathcal{M} \text { with } \int_{A} \gamma d x<\delta .
$$

Proof of Lemma 3.12. (i) We claim that $\left(\frac{\partial}{\partial x_{i}} u_{\epsilon}\right)_{\epsilon \in \mathbb{N}}$ is Cauchy in measure. Indeed, let $s>0$, consider

$A_{n, m}:=\left\{\left|\frac{\partial}{\partial x_{i}} u_{n}\right|>h\right\} \cup\left\{\left|\frac{\partial}{\partial x_{i}} u_{m}\right|>h\right\}, B_{n, m}:=\left\{\left|u_{n}-u_{m}\right|>k\right\}$ and $C_{n, m}:=\left\{\left|\frac{\partial}{\partial x_{i}} u_{n}\right| \leq h,\left|\frac{\partial}{\partial x_{i}} u_{m}\right| \leq h,\left|u_{n}-u_{m}\right| \leq k,\left|\frac{\partial}{\partial x_{i}} u_{n}-\frac{\partial}{\partial x_{i}} u_{m}>s\right|\right\}$, where $h$ and $k$ will be chosen later. One has

$$
\left\{\left|\frac{\partial}{\partial x_{i}} u_{n}-\frac{\partial}{\partial x_{i}} u_{m}\right|>s\right\} \subset A_{n, m} \cup B_{n, m} \cup C_{n, m} .
$$

Let $\vartheta>0$. By Lemma 3.9, we can choose $h=h(\vartheta)$ large enough such that meas $\left(A_{n, m}\right) \leq \frac{\vartheta}{3}$ for all $n, m \geq 0$. On the other hand, by Proposition 3.10, we have that meas $\left(B_{n, m}\right) \leq \frac{\vartheta}{3}$ 
for all $n, m \geq n_{0}(k, \vartheta)$. Moreover, by assumption $\left(H_{3}\right)$, there exists a real valued function $\gamma: \Omega \rightarrow[0, \infty]$ such that meas $\{x \in \Omega: \gamma(x)=0\}=0$ and

$$
\left(a_{i}(x, \xi)-a_{i}\left(x, \xi^{\prime}\right)\right) \cdot\left(\xi-\xi^{\prime}\right) \geq \gamma(x)
$$

for all $i=1, \ldots, N,|\xi|,\left|\xi^{\prime}\right| \leq h,\left|\xi-\xi^{\prime}\right| \geq s$, for a.e. $x \in \Omega$. Indeed, let's set $K=\{(\xi, \eta) \in$ $\mathbb{R} \times \mathbb{R}:|\xi| \leq h,|\eta| \leq h,|\xi-\eta| \geq s\}$. We have $K \subset B(0, h) \times B(0, h)$ and so $K$ is a compact set because it is closed in a compact set.

For all $x \in \Omega$ and for all $i=1, \ldots, N$, let us define $\psi: K \rightarrow[0 ; \infty]$ such that

$$
\psi(\xi, \eta)=\left(a_{i}(x, \xi)-a_{i}(x, \eta)\right) \cdot(\xi-\eta) .
$$

As for a.e. $x \in \Omega, a_{i}(x,$.$) is continuous on \mathbb{R}, \psi$ is continuous on the compact $K$, by Weierstrass theorem, there exists $\left(\xi_{0}, \eta_{0}\right) \in K$ such that

$$
\forall(\xi, \eta) \in K, \psi(\xi, \eta) \geq \psi\left(\xi_{0}, \eta_{0}\right)
$$

Now let us define $\gamma$ on $\Omega$ as follows.

$$
\gamma(x)=\psi_{i}\left(\xi_{0}, \eta_{0}\right)=\left(a_{i}\left(x, \xi_{0}\right)-a_{i}(x, \eta)\right) \cdot\left(\xi-\eta_{0}\right) .
$$

Since $s>0$, the function $\gamma$ is such that meas $(\{x \in \Omega: \gamma(x)=0\})=0$. Let $\delta=\delta(\epsilon)$ be given by Lemma 3.15, replacing $\epsilon$ and $A$ by $\frac{\epsilon}{3}$ and $C_{n, m}$ respectively. Taking respectively $\tilde{\xi}=T_{k}\left(u_{n}-u_{m}\right)$ and $\tilde{\xi}=T_{k}\left(u_{m}-u_{n}\right)$ for the weak solutions $u_{n}$ and $u_{m}$ in (3.19) and after adding the two relations, we have

$$
\left\{\begin{array}{l}
\sum_{i=1}^{N} \int_{\left\{\left|u_{n}-u_{m}\right|<k\right\}}\left(a_{i}\left(x, \frac{\partial}{\partial x_{i}} u_{n}\right)-a_{i}\left(x, \frac{\partial}{\partial x_{i}} u_{m}\right)\right)\left(\frac{\partial}{\partial x_{i}}\left(u_{n}-u_{m}\right)\right) d x \\
+\int_{Q}\left(\left(\frac{1}{\epsilon^{p_{i}(x)}}\left|\frac{\partial u_{n}}{\partial x_{i}}\right|^{p_{i}(x)-2} \frac{\partial u_{n}}{\partial x_{i}}\right)-\left(\frac{1}{\epsilon^{p_{i}(x)}}\left|\frac{\partial u_{m}}{\partial x_{i}}\right|^{p_{i}(x)-2} \frac{\partial u_{m}}{\partial x_{i}}\right)\right)\left(\frac{\partial\left(u_{n}-u_{m}\right)}{\partial x_{i}}\right) d x \\
+\int_{\Omega}\left(\left|u_{n}\right|^{p_{M}(x)-2} u_{n}-\left.u_{m}\right|^{p_{M}(x)-2} u_{m}\right)\left(T_{k}\left(u_{n}-u_{m}\right) d x+\int_{\tilde{\Gamma}_{N e}}\left(\tilde{\rho}\left(u_{n}\right)-\tilde{\rho}\left(u_{m}\right)\right) T_{k}\left(u_{n}-u_{m}\right) d \sigma\right. \\
=2\left(\int_{\Omega} T_{k}\left(u_{n}-u_{m}\right) d \mu_{\epsilon}+\int_{\tilde{\Gamma}_{N e}} \tilde{d}_{\epsilon} T_{k}\left(u_{n}-u_{m}\right) d \sigma\right),
\end{array}\right.
$$

where $Q=\left\{\tilde{\Omega} \backslash \Omega \cap\left\{\left|u_{n}-u_{m}\right|<k\right\}\right\}$. As the three last terms on the left hand side are non-negative and

$$
\int_{\Omega} T_{k}\left(u_{n}-u_{m}\right) d \mu_{\epsilon}+\int_{\tilde{\Gamma}_{N e}} \tilde{d}_{\epsilon} T_{k}\left(u_{n}-u_{m}\right) d \sigma \leq k\left(|\mu|(\Omega)+\|\tilde{d}\|_{L^{1}\left(\tilde{\Gamma}_{N e}\right)}\right),
$$

we deduce that

$\sum_{i=1}^{N} \int_{\left\{\left|u_{n}-u_{m}\right|<k\right\}}\left(a_{i}\left(x, \frac{\partial u_{n}}{\partial x_{i}}\right)-a_{i}\left(x, \frac{\partial u_{m}}{\partial x_{i}}\right)\right)\left(\frac{\partial\left(u_{n}-u_{m}\right)}{\partial x_{i}}\right) d x \leq 2 k\left(|\mu|(\Omega)+\|\tilde{d}\|_{L^{1}\left(\tilde{\Gamma}_{N e}\right)}\right)$. 
Therefore, using $\left(H_{3}\right)$ we have

$$
\begin{aligned}
\int_{C_{n, m}} \gamma d x & \leq \int_{C_{n, m}}\left(a_{i}\left(x, \frac{\partial}{\partial x_{i}} u_{n}\right)-a_{i}\left(x, \frac{\partial}{\partial x_{i}} u_{m}\right)\right) \frac{\partial}{\partial x_{i}}\left(u_{n}-u_{m}\right) d x \\
& \leq \sum_{i=1}^{N} \int_{C_{n, m}}\left(a_{i}\left(x, \frac{\partial}{\partial x_{i}} u_{n}\right)-a_{i}\left(x, \frac{\partial}{\partial x_{i}} u_{m}\right)\right) \frac{\partial}{\partial x_{i}}\left(u_{n}-u_{m}\right) d x \\
& \leq 2 k\left(\|\tilde{d}\|_{L^{1}\left(\tilde{\Gamma}_{N e}\right)}+|\mu|(\Omega)\right)<\delta
\end{aligned}
$$

by choosing $k=\delta / 4\left(\|\tilde{d}\|_{L^{1}\left(\tilde{\Gamma}_{N e}\right)}+|\mu|(\Omega)\right)$. From Lemma 3.15 again, it follows that meas $\left(C_{n, m}\right)<\frac{\vartheta}{3}$. Thus, using (3.35) and the estimates obtained for $A_{n, m}, B_{n, m}$ and $C_{n, m}$, it follows that

$$
\operatorname{meas}\left(\left\{\left|\frac{\partial}{\partial x_{i}} u_{n}-\frac{\partial}{\partial x_{i}} u_{m}\right|>s\right\}\right) \leq \vartheta
$$

for all $n, m \geq n_{0}(s, \vartheta)$, and then the claim is proved.

As consequence, $\left(\frac{\partial}{\partial x_{i}} u_{\epsilon}\right)_{\epsilon \in \mathbb{N}}$ converges in measure to some measurable function $\nu_{i}$.

In order to end the proof of Lemma 3.12, we need the following lemma.

Lemma 3.16. (a) For a.e. $k \in \mathbb{R}, \frac{\partial}{\partial x_{i}} T_{k}\left(u_{\epsilon}\right)$ converges in measure to $\nu_{i} \chi_{\{|u|<k\}}$.

(b) For a.e. $k \in \mathbb{R}, \frac{\partial}{\partial x_{i}} T_{k}(u)=\nu_{i} \chi_{\{|u|<k\}}$.

(c) $\frac{\partial}{\partial x_{i}} T_{k}(u)=\nu_{i} \chi_{\{|u|<k\}}$ holds for all $k \in \mathbb{R}$.

Proof. (a) We know that $\frac{\partial}{\partial x_{i}} u_{\epsilon} \rightarrow \nu_{i}$ in measure. Thus $\frac{\partial}{\partial x_{i}} u_{\epsilon} \chi_{\{|u|<k\}} \rightarrow \nu_{i} \chi_{\{|u|<k\}}$ in measure.

Now, let us show that $\left(\chi_{\left\{\left|u_{\epsilon}\right|<k\right\}}-\chi_{\{|u|<k\}}\right) \frac{\partial}{\partial x_{i}} u_{\epsilon} \rightarrow 0$ in measure.

For that, it is sufficient to show that $\left(\chi_{\left\{\left|u_{\epsilon}\right|<k\right\}}-\chi_{\{|u|<k\}}\right) \rightarrow 0$ in measure. Now, for all $\delta>0,\left\{\left|\chi_{\left\{\left|u_{\epsilon}\right|<k\right\}}-\chi_{\{|u|<k\}}\right|\left|\frac{\partial}{\partial x_{i}} u_{\epsilon}\right|>\delta\right\} \subset\left\{\left|\chi_{\left\{\left|u_{\epsilon}\right|<k\right\}}-\chi_{\{|u|<k\}}\right| \neq 0\right\} \subset\{|u|=$ $k\} \cup\left\{u_{\epsilon}<k<u\right\} \cup\left\{u<k<u_{\epsilon}\right\} \cup\left\{u_{\epsilon}<-k<u\right\} \cup\left\{u<-k<u_{\epsilon}\right\}$. Thus,

$$
\left\{\begin{array}{l}
\quad \text { meas }\left(\left\{\left|\chi_{\left\{\left|u_{\epsilon}\right|<k\right\}}-\chi_{\{|u|<k\}}\right|\left|\frac{\partial}{\partial x_{i}} u_{\epsilon}\right|>\delta\right\}\right) \\
\quad \text { meas }(\{|u|=k\})+\text { meas }\left(\left\{u_{\epsilon}<k<u\right\}\right) \\
+ \text { meas }\left(\left\{u<k<u_{\epsilon}\right\}\right) \\
+ \text { meas }\left(\left\{u_{\epsilon}<-k<u\right\}\right) \\
+ \text { meas }\left(\left\{u<-k<u_{\epsilon}\right\}\right) .
\end{array}\right.
$$

Note that

meas $(\{|u|=k\}) \leq$ meas $(\{k-h<u<k+h\})+$ meas $(\{-k-h<u<-k+h\}) \rightarrow 0$ as $h \rightarrow 0$ for a.e. $k>0$, since $u$ is fixed function.

Next, meas $\left(\left\{u_{\epsilon}<k<u\right\}\right) \leq$ meas $(\{k<u<k+h\})+$ meas $\left(\left\{\left|u_{\epsilon}-u\right|>h\right\}\right)$, for all 
$h>0$.

Due to Proposition 3.10, we have for all fixed $h>0$, meas $\left(\left\{\left|u_{\epsilon}-u\right|>h\right\}\right) \rightarrow 0$ as $\epsilon \rightarrow 0$. Since meas $(\{k<u<k+h\}) \rightarrow 0$ as $h \rightarrow 0$, for all $\vartheta>0$, one can find $N$ such that for all $n>N$, meas $(\{|u|=k\})<\frac{\vartheta}{2}+\frac{\vartheta}{2}=\vartheta$ by choosing $h$ and then $N$. Each of the other terms on the right-hand side of (3.37) can be treated in the same way as for meas $\left(\left\{u_{\epsilon}<k<u\right\}\right)$. Thus, meas $\left.\left(\left\{\left|\chi_{\left\{\left|u_{\epsilon}\right|<k\right\}}-\chi_{\{|u|<k\}}\right|\left|\frac{\partial}{\partial x_{i}} u_{\epsilon}\right|>\delta\right\}\right\}\right) \rightarrow 0$ as $\epsilon \rightarrow 0$. Finally, since $\frac{\partial}{\partial x_{i}} T_{k}\left(u_{\epsilon}\right)=\frac{\partial}{\partial x_{i}} u_{\epsilon} \chi_{\left\{\left|u_{\epsilon}\right|<k\right\}}$, the claim (a) follows.

(b) Using the Proof of Proposition 3.10-(ii) we have $\frac{\partial}{\partial x_{i}} T_{k}\left(u_{\epsilon}\right) \rightarrow \frac{\partial}{\partial x_{i}} T_{k}(u)$ weakly in $L^{p_{i}^{-}}(\tilde{\Omega})$. The previous convergence also ensures that $\frac{\partial}{\partial x_{i}} T_{k}\left(u_{\epsilon}\right)$ converges to $\frac{\partial}{\partial x_{i}} T_{k}(u)$ weakly in $L^{1}(\Omega)$. On the other hand, by $(a), \frac{\partial}{\partial x_{i}} T_{k}\left(u_{\epsilon}\right)$ converges to $\nu_{i} \chi_{\{|u|<k\}}$ in measure. By Lemma 3.14, since $\frac{\partial}{\partial x_{i}} T_{k}\left(u_{\epsilon}\right)$ is uniformly bounded in $L^{p^{-}}(\tilde{\Omega})$ (see Lemma 3.8 ) hence in $L^{p_{i}^{-}}(\Omega)$, the convergence is actually strong in $L^{1}(\Omega)$; thus it is also weak in $L^{1}(\Omega)$. By the uniqueness of the weak $L^{1}$-limit, $\nu_{i} \chi_{\{|u|<k\}}$ coincides with $\frac{\partial}{\partial x_{i}} T_{k}(u)$.

(c) Let $0<k<s$, and $s$ be such that $\nu_{i} \chi_{\{|u|<s\}}$ coincides with $\frac{\partial}{\partial x_{i}} T_{s}(u)$. Then,

$$
\begin{aligned}
\frac{\partial}{\partial x_{i}} T_{k}(u) & =\frac{\partial}{\partial x_{i}} T_{k}\left(T_{s}(u)\right) \\
& =\frac{\partial}{\partial x_{i}} T_{s}(u) \chi_{\left\{\left|T_{s}(u)\right|<k\right\}} \\
& =\nu_{i} \chi_{\{|u|<s\}} \chi_{\{|u|<k\}} \\
& =\nu_{i} \chi_{\{|u|<k\} .} .
\end{aligned}
$$

Now, we can end the proof of Lemma 3.12. Indeed, combining lemmas 3.16 (c) and 3.13; (i) follows.

Next, by lemmas 3.14 and 3.16 , we have for all $k>0, i=1, \ldots, N, a_{i}\left(x, \frac{\partial}{\partial x_{i}} T_{k}\left(u_{\epsilon}\right)\right)$ converges to $a_{i}\left(x, \frac{\partial}{\partial x_{i}} T_{k}(u)\right)$ in $L^{1}(\Omega)$ strongly. Indeed, let $s, k>0$, consider $E_{4}=\left\{\left|\frac{\partial u_{n}}{\partial x_{i}}-\frac{\partial u_{m}}{\partial x_{i}}\right|>s,\left|u_{n}\right| \leq k,\left|u_{m}\right| \leq k\right\}, E_{5}=\left\{\left|\frac{\partial u_{m}}{\partial x_{i}}\right|>s,\left|u_{n}\right|>k,\left|u_{m}\right| \leq k\right\}, E_{6}=$ $\left\{\left|\frac{\partial u_{n}}{\partial x_{i}}\right|>s,\left|u_{n}\right| \leq k,\left|u_{m}\right|>k\right\}$.

We have

$$
\left\{\left|\frac{\partial T_{k}\left(u_{n}\right)}{\partial x_{i}}-\frac{\partial T_{k}\left(u_{m}\right)}{\partial x_{i}}\right|>s\right\} \subset E_{4} \cup E_{5} \cup E_{6}
$$


$\forall \vartheta>0$, by Lemma 3.7, there exists $k(\vartheta)$ such that

$$
\operatorname{meas}\left(E_{5}\right) \leq \frac{\vartheta}{3} \text { and } \operatorname{meas}\left(E_{6}\right) \leq \frac{\vartheta}{3} .
$$

Using (3.36)-(3.39), we get

$$
\text { meas }\left(\left\{\left|\frac{\partial}{\partial x_{i}} T_{k}\left(u_{n}\right)-\frac{\partial}{\partial x_{i}} T_{k}\left(u_{m}\right)\right|>s\right\}\right) \leq \vartheta,
$$

for all $n, m \geq n_{1}(s, \vartheta)$. Therefore, $\frac{\partial T_{k}\left(u_{\epsilon}\right)}{\partial x_{i}}$ converges in measure to $\frac{\partial T_{k}(u)}{\partial x_{i}}$. Using lemmas 3.8 and 3.14 , we deduce that $\frac{\partial T_{k}\left(u_{\epsilon}\right)}{\partial x_{i}}$ converges to $\frac{\partial T_{k}(u)}{\partial x_{i}}$ in $L^{1}(\Omega)$. So, after passing to a suitable subsequence of $\left(\frac{\partial T_{k}\left(u_{\epsilon}\right)}{\partial x_{i}}\right)_{\epsilon>0}$, we can assume that $\frac{\partial T_{k}\left(u_{\epsilon}\right)}{\partial x_{i}}$ converges to $\frac{\partial T_{k}(u)}{\partial x_{i}}$ a.e. in $\Omega$. By the continuity of $a_{i}(x,$.$) , we deduce that a_{i}\left(x, \frac{\partial T_{k}\left(u_{\epsilon}\right)}{\partial x_{i}}\right)$ converges to $a_{i}\left(x, \frac{\partial T_{k}(u)}{\partial x_{i}}\right)$ a.e. in $\Omega$. As $\Omega$ is bounded, this convergence is in measure. Using lemmas 3.14 and 3.16 , we deduce that for all $k>0, i=1, \ldots, N, a_{i}\left(x, \frac{\partial}{\partial x_{i}} T_{k}\left(u_{\epsilon}\right)\right)$ converges to $a_{i}\left(x, \frac{\partial}{\partial x_{i}} T_{k}(u)\right)$ in $L^{1}(\Omega)$ strongly and $a_{i}\left(x, \frac{\partial}{\partial x_{i}} T_{k}\left(u_{\epsilon}\right)\right)$ converges to $\chi_{k} \in L^{p_{i}^{\prime}(\cdot)}(\Omega)$ weakly in $L^{p_{i}^{\prime}(.)}(\Omega)$. Since each of the convergences implies the weak $L^{1}$-convergence, $\chi_{k}$ can be identified with $a_{i}\left(x, \frac{\partial}{\partial x_{i}} T_{k}(u)\right)$; thus, $a_{i}\left(x, \frac{\partial}{\partial x_{i}} T_{k}(u)\right) \in L^{p_{i}^{\prime}(\cdot)}(\Omega)$

By using Lebesgue generalized convergence theorem and above results, we deduce the following result.

Proposition 3.17. For any $k>0$ and any $i=1, \ldots, N$, as $\epsilon$ tends to 0 , we have

(i) $\frac{\partial T_{k}\left(u_{\epsilon}\right)}{\partial x_{i}} \rightarrow \frac{\partial T_{k}(u)}{\partial x_{i}}$ a.e. in $\Omega$,

(ii) $a_{i}\left(x, \frac{\partial T_{k}\left(u_{\epsilon}\right)}{\partial x_{i}}\right) \frac{\partial T_{k}\left(u_{\epsilon}\right)}{\partial x_{i}} \rightarrow a_{i}\left(x, \frac{\partial T_{k}(u)}{\partial x_{i}}\right) \frac{\partial T_{k}(u)}{\partial x_{i}}$ a.e. in $\Omega$ and strongly in $L^{1}(\Omega)$,

(iii) $\frac{\partial T_{k}\left(u_{\epsilon}\right)}{\partial x_{i}} \rightarrow \frac{\partial T_{k}(u)}{\partial x_{i}}$ strongly in $L^{p_{i}(x)}(\Omega)$.

\section{Existence and uniqueness of solution to $P(\rho, \mu, d)$}

We are now able to prove Theorem 2.6.

\section{Proof of Theorem 2.6}

Thanks to the Proposition 3.10 and as $\forall k>0, \forall i=1, \ldots, N, \frac{\partial T_{k}(u)}{\partial x_{i}}=0$ in $L^{p_{i}^{-}}(\tilde{\Omega} \backslash \Omega)$, then, $\forall k>0, T_{k}(u)=$ constant a.e. on $\tilde{\Omega} \backslash \Omega$. Hence, we conclude that $u \in \mathcal{T}_{N e}^{1, \vec{p}(.)}(\Omega)$. 
We already state that $b(u) \in L^{1}(\Omega)$.

To show that $u$ is an entropy solution of $P(\rho, \mu, d)$, we only have to prove the inequality in (2.9). Let $\varphi \in W_{D}^{1, \vec{p}(.)}(\Omega) \cap L^{\infty}(\Omega)$. We consider the function $\varphi_{1} \in W_{D}^{1, \vec{p}(.)}(\tilde{\Omega}) \cap L^{\infty}(\Omega)$ such that

$$
\varphi_{1}=\varphi \chi_{\Omega}+\varphi_{N} \chi_{\Omega} \backslash \Omega
$$

We set $\tilde{\xi}=T_{k}\left(u_{\epsilon}-\varphi_{1}\right)$ in (3.19) to get

$$
\left\{\begin{array}{l}
\sum_{i=1}^{N} \int_{\Omega}\left(a_{i}\left(x, \frac{\partial}{\partial x_{i}} u_{\epsilon}\right) \cdot \frac{\partial}{\partial x_{i}} T_{k}\left(u_{\epsilon}-\varphi\right)\right) d x \\
+\sum_{i=1}^{N} \int_{\tilde{\Omega} \backslash \Omega}\left(\frac{1}{\epsilon^{p_{i}(x)}}\left|\frac{\partial}{\partial x_{i}} u_{\epsilon}\right|^{p_{i}(x)-2} \frac{\partial}{\partial x_{i}} u_{\epsilon} \cdot \frac{\partial}{\partial x_{i}} T_{k}\left(u_{\epsilon}-\varphi_{N}\right)\right) d x \\
\int_{\Omega} b\left(u_{\epsilon}\right) T_{k}\left(u_{\epsilon}-\varphi\right) d x=\int_{\Omega} T_{k}\left(u_{\epsilon}-\varphi\right) d \mu_{\epsilon}+\int_{\tilde{\Gamma}_{N e}}\left(\tilde{d}_{\epsilon}-\tilde{\rho}\left(u_{\epsilon}\right)\right) T_{k}\left(u_{\epsilon}-\varphi_{N}\right) d \sigma .
\end{array}\right.
$$

The following convergence result hold true.

Lemma 4.1. For any $k>0$, for all $i=1, \ldots, N$, as $\epsilon \rightarrow 0$,

$$
\frac{\partial}{\partial x_{i}} T_{k}\left(u_{\epsilon}-\varphi\right) \rightarrow \frac{\partial}{\partial x_{i}} T_{k}(u-\varphi) \text { strongly in } L^{p_{i}(.)}(\Omega)
$$

Proof. Let $k>0, i=1, \ldots, N$. We have

$$
\begin{aligned}
& \int_{\Omega}\left|\frac{\partial}{\partial x_{i}} T_{k}\left(u_{\epsilon}-\varphi\right)-\frac{\partial}{\partial x_{i}} T_{k}(u-\varphi)\right|^{p_{i}(x)} d x \\
= & \int_{\Omega \cap\left[\left|u_{\epsilon}-\varphi\right| \leq k,|u-\varphi| \leq k\right]}\left|\frac{\partial}{\partial x_{i}} u_{\epsilon}-\frac{\partial}{\partial x_{i}} u\right|^{p_{i}(x)} d x \\
\leq & \int_{\Omega \cap\left[\left|u_{\epsilon}\right| \leq l,|u| \leq l\right]}\left|\frac{\partial u_{\epsilon}}{\partial x_{i}}-\frac{\partial u}{\partial x_{i}}\right|^{p_{i}(x)} d x, \text { with } l=k+\|\varphi\|_{\infty} \\
= & \int_{\Omega}\left|\frac{\partial}{\partial x_{i}} T_{l}\left(u_{\epsilon}\right)-\frac{\partial}{\partial x_{i}} T_{l}(u)\right|^{p_{i}(x)} d x \\
\rightarrow & 0 \text { as } \epsilon \rightarrow 0 \text { by Proposition } 3.17-(i i i) .
\end{aligned}
$$

We need to pass to the limit in (4.1) as $\epsilon \rightarrow 0$. We have

$$
\sum_{i=1}^{N} \int_{\Omega}\left(a_{i}\left(x, \frac{\partial}{\partial x_{i}} u_{\epsilon}\right) \frac{\partial}{\partial x_{i}} T_{k}\left(u_{\epsilon}-\varphi\right)\right) d x=\sum_{i=1}^{N} \int_{\Omega}\left(a_{i}\left(x, \frac{\partial T_{l}\left(u_{\epsilon}\right)}{\partial x_{i}}\right) \frac{\partial}{\partial x_{i}} T_{k}\left(u_{\epsilon}-\varphi\right)\right) d x
$$

with $l=k+\|\varphi\|_{\infty}$, then, by Lemma 3.12- (ii) and Lemma 4.1, we have

$$
\lim _{\epsilon \rightarrow 0} \sum_{i=1}^{N} \int_{\Omega}\left(a_{i}\left(x, \frac{\partial T_{l}\left(u_{\epsilon}\right)}{\partial x_{i}}\right) \frac{\partial}{\partial x_{i}} T_{k}\left(u_{\epsilon}-\varphi\right)\right) d x=\sum_{i=1}^{N} \int_{\Omega}\left(a_{i}\left(x, \frac{\partial T_{l}(u)}{\partial x_{i}}\right) \frac{\partial}{\partial x_{i}} T_{k}(u-\varphi)\right) d x ;
$$

that is

$$
\lim _{\epsilon \rightarrow 0} \sum_{i=1}^{N} \int_{\Omega}\left(a_{i}\left(x, \frac{\partial}{\partial x_{i}} u_{\epsilon}\right) \frac{\partial}{\partial x_{i}} T_{k}\left(u_{\epsilon}-\varphi\right)\right) d x=\sum_{i=1}^{N} \int_{\Omega}\left(a_{i}\left(x, \frac{\partial T_{l}(u)}{\partial x_{i}}\right) \frac{\partial}{\partial x_{i}} T_{k}(u-\varphi)\right) d x .
$$


For the second term in the left hand side of (4.1), we have

$$
\limsup _{\epsilon \rightarrow 0} \sum_{i=1}^{N} \int_{\tilde{\Omega} \backslash \Omega}\left(\frac{1}{\epsilon^{p_{i}(x)}}\left|\frac{\partial}{\partial x_{i}} u_{\epsilon}\right|^{p_{i}(x)-2} \frac{\partial}{\partial x_{i}} u_{\epsilon} \frac{\partial}{\partial x_{i}} T_{k}\left(u_{\epsilon}-\varphi_{N}\right)\right) d x \geq 0 .
$$

Indeed

$$
\left\{\begin{aligned}
& \sum_{i=1}^{N} \int_{\tilde{\Omega} \backslash \Omega}\left(\frac{1}{\epsilon^{p_{i}(x)}}\left|\frac{\partial}{\partial x_{i}} u_{\epsilon}\right|^{p_{i}(x)-2} \frac{\partial}{\partial x_{i}} u_{\epsilon} \frac{\partial}{\partial x_{i}} T_{k}\left(u_{\epsilon}-\varphi_{N}\right)\right) d x \\
= & \sum_{i=1}^{N} \int_{\tilde{\Omega} \backslash \Omega \cap\left[\left|u_{\epsilon}-\varphi\right| \leq k\right]}\left(\frac{1}{\epsilon^{p_{i}(x)}}\left|\frac{\partial}{\partial x_{i}} u_{\epsilon}\right|^{p_{i}(x)-2} \frac{\partial}{\partial x_{i}} u_{\epsilon} \frac{\partial}{\partial x_{i}}\left(u_{\epsilon}-\varphi_{N}\right)\right) d x \\
= & \sum_{i=1}^{N} \int_{\tilde{\Omega} \backslash \Omega \cap\left[\left|u_{\epsilon}-\varphi\right| \leq k\right]}\left(\frac{1}{\epsilon^{p_{i}(x)}}\left|\frac{\partial}{\partial x_{i}} u_{\epsilon}\right|^{p_{i}(x)}\right) d x \geq 0 .
\end{aligned}\right.
$$

Hence, we get (4.3).

Let us examine the last term in the left hand side of (4.1).

we have

$$
\int_{\Omega} b\left(u_{\epsilon}\right) T_{k}\left(u_{\epsilon}-\varphi\right) d x=\int_{\Omega}\left(b\left(u_{\epsilon}\right)-b(\varphi)\right) T_{k}\left(u_{\epsilon}-\varphi\right) d x+\int_{\Omega} b(\varphi) T_{k}\left(u_{\epsilon}-\varphi\right) d x .
$$

As $b$ is non-decreasing,

$$
\left(b\left(u_{\epsilon}\right)-b(\varphi)\right) T_{k}\left(u_{\epsilon}-\varphi\right) \geq 0 \text { a.e. in } \Omega
$$

and we get by Fatou's lemma that

$$
\liminf _{\epsilon \rightarrow 0} \int_{\Omega}\left(b\left(u_{\epsilon}\right)-b(\varphi)\right) T_{k}\left(u_{\epsilon}-\varphi\right) d x \geq \int_{\Omega}(b(u)-b(\varphi)) T_{k}(u-\varphi) d x .
$$

As $\varphi \in L^{\infty}(\Omega)$, we obtain $b(\varphi) \in L^{\infty}(\Omega)$ and so $b(\varphi) \in L^{1}(\Omega)$ (as $\Omega$ is bounded) and by Lebesgue dominated convergence theorem, we deduce that

$$
\lim _{\epsilon \rightarrow 0} \int_{\Omega} b(\varphi) T_{k}\left(u_{\epsilon}-\varphi\right) d x=\int_{\Omega} b(\varphi) T_{k}(u-\varphi) d x
$$

Consequently,

$$
\limsup _{\epsilon \rightarrow 0} \int_{\Omega} b\left(u_{\epsilon}\right) T_{k}\left(u_{\epsilon}-\varphi\right) d x \geq \int_{\Omega} b(u) T_{k}(u-\varphi) d x .
$$

As $f_{\epsilon} \rightarrow f$ strongly in $L^{1}(\Omega)$ and $T_{k}\left(u_{\epsilon}-v\right) \rightarrow^{*} T_{k}(u-v)$ in $L^{\infty}(\Omega)$, using the Lebesgue generalized convergence theorem we have

$$
\left\{\begin{array}{l}
\lim _{\epsilon \rightarrow 0} \int_{\Omega} f_{\epsilon} T_{k}\left(u_{\epsilon}-\varphi\right) d x=\int_{\Omega} T_{k}(u-\varphi) d x \\
\lim _{\epsilon \rightarrow 0} \int_{\tilde{\Gamma}_{N e}} \tilde{d}_{\epsilon} T_{k}\left(u_{\epsilon}-\varphi_{N}\right) d \sigma=\int_{\Omega} \tilde{d} T_{k}\left(u-\varphi_{N}\right) d \sigma .
\end{array}\right.
$$

Since $\nabla T_{k}\left(u_{\epsilon}-\varphi\right) \rightarrow \nabla T_{k}(u-\varphi)$ in $\left(L^{p_{m}(.)}(\Omega)\right)^{N}$ and $F \in\left(L^{p_{m}^{\prime}(.)}(\Omega)\right)^{N}$

$$
\lim _{\epsilon \rightarrow 0} \int_{\Omega} F \cdot \nabla T_{k}\left(u_{\epsilon}-\varphi\right) d x=\int_{\Omega} F . \nabla T_{k}(u-\varphi) d x .
$$

We know that $\forall k>0, T_{k}(u)=$ constant on $\tilde{\Omega} \backslash \Omega$, then, it yields that $u=$ constant on $\tilde{\Omega} \backslash \Omega$. So, one has

$$
\lim _{\epsilon \rightarrow 0} \int_{\tilde{\Gamma}_{N e}} \tilde{d}_{\epsilon} T_{k}\left(u_{\epsilon}-\varphi\right) d x=d T_{k}\left(u_{N}-\varphi_{N}\right) .
$$


At last, we have

$$
\int_{\tilde{\Gamma}_{N e}} \tilde{\rho}\left(u_{\epsilon}\right) T_{k}\left(u_{\epsilon}-\varphi_{N}\right) d \sigma=\int_{\tilde{\Gamma}_{N e}}\left(\tilde{\rho}\left(u_{\epsilon}\right)-\tilde{\rho}\left(\varphi_{N}\right)\right) T_{k}\left(u_{\epsilon}-\varphi_{N}\right) d \sigma+\int_{\tilde{\Gamma}_{N e}} \tilde{\rho}\left(\varphi_{N}\right) T_{k}\left(u_{\epsilon}-\varphi_{N}\right) d \sigma .
$$

As $\tilde{\rho}$ is non-decreasing,

$$
\left(\tilde{\rho}\left(u_{\epsilon}\right)-\tilde{\rho}\left(\varphi_{N}\right)\right) T_{k}\left(u_{\epsilon}-\varphi_{N}\right) \geq 0 \text { a.e. in } \tilde{\Gamma}_{N e}
$$

and we get by Fatou's lemma that

$$
\begin{aligned}
\liminf _{\epsilon \rightarrow 0} \int_{\tilde{\Gamma}_{N e}}\left(\tilde{\rho}\left(u_{\epsilon}\right)-\tilde{\rho}\left(\varphi_{N}\right)\right) T_{k}\left(u_{\epsilon}-\varphi_{N}\right) d \sigma & \geq \int_{\tilde{\Gamma}_{N e}}\left(\tilde{\rho}\left(u_{N}\right)-\tilde{\rho}\left(\varphi_{N}\right)\right) T_{k}\left(u_{N}-\varphi_{N}\right) d \sigma \\
& =\left(\rho\left(u_{N}\right)-\rho\left(\varphi_{N}\right)\right) T_{k}\left(u_{N}-\varphi_{N}\right) .
\end{aligned}
$$

As $\varphi_{N} \in L^{\infty}\left(\tilde{\Gamma}_{N e}\right)$, we obtain $\tilde{\rho}\left(\varphi_{N}\right) \in L^{\infty}\left(\tilde{\Gamma}_{N e}\right)$ and so $\tilde{\rho}\left(\varphi_{N}\right) \in L^{1}\left(\tilde{\Gamma}_{N e}\right)$ (as $\tilde{\Gamma}_{N e}$ is bounded) and by the Lebesgue dominated convergence theorem, we deduce that

$$
\lim _{\epsilon \rightarrow 0} \int_{\tilde{\Gamma}_{N e}} \tilde{\rho}\left(\varphi_{N}\right) T_{k}\left(u_{\epsilon}-\varphi_{N}\right) d \sigma=\int_{\tilde{\Gamma}_{N e}} \tilde{\rho}\left(\varphi_{N}\right) T_{k}\left(u_{N}-\varphi_{N}\right) d \sigma=\rho\left(\varphi_{N}\right) T_{k}\left(u_{N}-\varphi_{N}\right) .
$$

Hence,

$$
\limsup _{\epsilon \rightarrow 0} \int_{\tilde{\Gamma}_{N e}} \tilde{\rho}\left(u_{\epsilon}\right) T_{k}\left(u_{\epsilon}-\varphi_{N}\right) d \sigma \geq \rho\left(\varphi_{N}\right) T_{k}\left(u_{N}-\varphi_{N}\right)
$$

Passing to the limit as $\epsilon \rightarrow 0$ in (4.1) and using (4.2)-(4.8), we see that $u$ is an entropy solution of $P(\rho, \mu, d)$.

We now prove the uniqueness part of Theorem 2.6.

Let $u$ and $v$ be two entropy solutions of $P(\rho, \mu, d)$.

Let $h>0$. For $u$, we take $\xi=T_{h}(v)$ as test function and for $v$, we take $\xi=T_{h}(u)$ as test function in (2.9), to get for any $k>0$ with $k<h$,

$$
\left\{\begin{array}{l}
\int_{\Omega}\left(\sum_{i=1}^{N} a_{i}\left(x, \frac{\partial}{\partial x_{i}} u\right) \frac{\partial}{\partial x_{i}} T_{k}\left(u-T_{h}(v)\right)\right) d x+\int_{\Omega} b(u) T_{k}\left(u-T_{h}(v)\right) d x \leq \\
\int_{\Omega} f T_{k}\left(u-T_{h}(v)\right) d x+\int_{\Omega} F \cdot \nabla T_{k}\left(u-T_{h}(v)\right) d x+\left(d-\rho\left(u_{N e}\right)\right) T_{k}\left(u_{N e}-T_{h}(v)\right)
\end{array}\right.
$$

and

$$
\left\{\begin{array}{l}
\int_{\Omega}\left(\sum_{i=1}^{N} a_{i}\left(x, \frac{\partial}{\partial x_{i}} v\right) \frac{\partial}{\partial x_{i}} T_{k}\left(v-T_{h}(u)\right)\right) d x+\int_{\Omega} b(v) T_{k}\left(v-T_{h}(u)\right) d x \leq \\
\int_{\Omega} f T_{k}\left(v-T_{h}(u)\right) d x+\int_{\Omega} F \cdot \nabla T_{k}\left(v-T_{h}(u)\right) d x+\left(d-\rho\left(v_{N e}\right)\right) T_{k}\left(v_{N e}-T_{h}(u)\right) .
\end{array}\right.
$$


By adding (4.9) and (4.10), we obtain

$$
\begin{cases}\int_{\Omega}\left(\sum_{i=1}^{N} a_{i}\left(x, \frac{\partial}{\partial x_{i}} u\right) \frac{\partial}{\partial x_{i}} T_{k}\left(u-T_{h}(v)\right)\right) d x & \\ +\int_{\Omega}\left(\sum_{i=1}^{N} a_{i}\left(x, \frac{\partial}{\partial x_{i}} v\right) \frac{\partial}{\partial x_{i}} T_{k}\left(v-T_{h}(u)\right)\right) d x & :=A(h, k) \\ +\int_{\Omega} b(u) T_{k}\left(u-T_{h}(v)\right) d x+\int_{\Omega} b(v) T_{k}\left(v-T_{h}(u)\right) d x & :=B(h, k) \\ +\rho\left(u_{N e}\right) T_{k}\left(u_{N e}-T_{h}(v)\right)+\rho\left(v_{N e}\right) T_{k}\left(v_{N e}-T_{h}(u)\right) & :=C(h, k) \\ \leq \int_{\Omega} f T_{k}\left(u-T_{h}(v)\right) d x+\int_{\Omega} f T_{k}\left(v-T_{h}(u)\right) d x & :=D(h, k) \\ +\int_{\Omega} F \cdot \nabla T_{k}\left(u-T_{h}(v)\right) d x+\int_{\Omega} F \cdot \nabla T_{k}\left(v-T_{h}(u)\right) d x & :=T(h, k) \\ +d T_{k}\left(u_{N e}-T_{h}(v)\right)+d T_{k}\left(v_{N e}-T_{h}(u)\right) & :=E(h, k) .\end{cases}
$$

Let us introduce the following subsets of $\Omega$.

$$
\begin{aligned}
& A_{0}:=[|u-v|<k,|u|<h,|v|<h] \\
& A_{1}:=\left[\left|u-T_{h}(v)\right|<k,|v| \geq h\right] \\
& A_{1}^{\prime}:=\left[\left|v-T_{h}(u)\right|<k,|u| \geq h\right] \\
& A_{2}:=\left[\left|u-T_{h}(v)\right|<k,|u| \geq h,|v|<h\right] \\
& A_{2}^{\prime}:=\left[\left|v-T_{h}(u)\right|<k,|v| \geq h,|u|<h\right] .
\end{aligned}
$$

We have the following assertion (see [22] for the proof).

Assertion 4.2. If $u$ is an entropy solution of $P(\rho, \mu, d)$, then $A_{2} \subset F_{h, k}$ and $A_{1} \subset F_{h-k, 2 k}$, where

$$
F_{h, k}=\{h \leq|u|<h+k, h>0, k>0\} .
$$

Assertion 4.3. Let $u$ be an entropy solution of $P(\rho, \mu, d)$. On $A_{2}$ (and on $A_{1}$ ) we have according to Hölder inequality.

$$
\begin{gathered}
\int_{A_{2}} F \cdot \nabla u d x \leq\left(\int_{A_{2}}|F|^{\left(p_{m}^{\prime}\right)^{-}} d x\right)^{\frac{1}{\left(p_{m}^{\prime}\right)^{-}}}\left(\int_{A_{2}}|\nabla u|^{p_{m}^{-}}\right)^{\frac{1}{p_{m}^{-}}} d x, \\
\text { with } \lim _{h \rightarrow \infty}\left(\int_{A_{2}}|F|^{\left(p_{m}^{\prime}\right)^{-}} d x\right)^{\frac{1}{\left(p_{m}^{\prime}\right)^{-}}}\left(\int_{A_{2}}|\nabla u|^{p_{m}^{-}} d x\right)^{\frac{1}{p_{m}^{-}}}=0 .
\end{gathered}
$$

(2)

$$
\begin{gathered}
\int_{A_{1}} F \cdot \nabla u d x \leq\left(\int_{A_{1}}|F|^{\left(p_{m}^{\prime}\right)^{-}} d x\right)^{\frac{1}{\left(p_{m}^{\prime}\right)^{-}}}\left(\int_{A_{1}}|\nabla u|^{p_{m}^{-}} d x\right)^{\frac{1}{p_{m}^{-}}}, \\
\text {with } \lim _{h \rightarrow \infty}\left(\int_{A_{1}}|F|^{\left(p_{m}^{\prime}\right)^{-}} d x\right)^{\frac{1}{\left(p_{m}^{\prime}\right)^{-}}}\left(\int_{A_{1}}|\nabla u|^{p_{m}^{-}} d x\right)^{\frac{1}{p_{m}^{-}}}=0 .
\end{gathered}
$$


Proof. (1) $\lim _{h \rightarrow \infty}\left(\int_{A_{2}}|F|^{\left(p_{m}^{\prime}\right)^{-}} d x\right)^{\frac{1}{\left(p_{m}^{\prime}\right)^{-}}}=0$ (see [22]).

Now, it remains to prove that $\left(\int_{A_{2}}|\nabla u|^{p_{m}^{-}} d x\right)^{\frac{1}{p_{m}^{-}}}$is bounded with respect to $h$.

We make the following notations:

$\mathcal{I}=\left\{i \in\{1, \ldots, N\}:\left\{\left|\frac{\partial}{\partial x_{i}} u\right|\right\} \leq 1\right\}$ and $\mathcal{J}=\left\{i \in\{1, \ldots, N\}:\left\{\left|\frac{\partial}{\partial x_{i}} u\right|\right\}>1\right\}$.

We have

$$
\begin{aligned}
\sum_{i=1}^{N} \int_{F_{h, k}}\left|\frac{\partial}{\partial x_{i}} u\right|^{p_{i}(x)} d x & =\sum_{i \in \mathcal{I}}\left(\int_{F_{h, k}}\left|\frac{\partial}{\partial x_{i}} u\right|^{p_{i}(x)} d x\right)+\sum_{i \in \mathcal{J}}\left(\int_{F_{h, k}}\left|\frac{\partial}{\partial x_{i}} u\right|^{p_{i}(x)} d x\right) \\
& \geq \sum_{i \in \mathcal{J}}\left(\int_{F_{h, k}}\left|\frac{\partial}{\partial x_{i}} u\right|^{p_{i}(x)} d x\right) \\
& \geq \sum_{i \in \mathcal{J}}\left(\int_{F_{h, k}}\left|\frac{\partial}{\partial x_{i}} u\right|^{p_{m}^{-}} d x\right) \\
& \geq \sum_{i=1}^{N}\left(\int_{F_{h, k}}\left|\frac{\partial}{\partial x_{i}} u\right|^{p_{m}^{-}} d x\right)-\sum_{i \in \mathcal{I}}\left(\int_{F_{h, k}}\left|\frac{\partial}{\partial x_{i}} u\right|^{p_{m}^{-}} d x\right) \\
& \geq \sum_{i=1}^{N}\left(\int_{F_{h, k}}\left|\frac{\partial}{\partial x_{i}} u\right|^{p_{m}^{-}}\right)-N \operatorname{Neas}(\Omega) \\
& \geq \sum_{i=1}^{N} \|\left.\frac{\partial}{\partial x_{i}} u\right|_{\left(L^{p_{m}^{-}}\left(F_{h, k}\right)\right)^{N}} ^{p_{m}^{-}}-\operatorname{Nmeas}(\Omega) \\
& \geq C\|\nabla u\|_{\left(L_{m}^{p_{m}}\left(F_{h, k}\right)\right)^{N}}^{p^{-}}-\operatorname{Nmeas}(\Omega) .
\end{aligned}
$$

We deduce that

$$
\sum_{i=1}^{N} \int_{F_{h, k}}\left|\frac{\partial}{\partial x_{i}} u\right|^{p_{i}(x)} d x \geq C \int_{F_{h, k}}|\nabla u|^{p_{m}^{-}} d x-\operatorname{Nmeas}(\Omega) .
$$

Choosing $T_{h}(u)$ as test function in (2.9), we get

$$
\left\{\begin{array}{l}
\left.\int_{\Omega}\left(\sum_{i=1}^{N} a_{i}\left(x, \frac{\partial}{\partial x_{i}} u\right)\right) \frac{\partial}{\partial x_{i}} T_{k}\left(u-T_{h}(u)\right)\right) d x+\int_{\Omega}|u|^{p_{M}(x)-2} u T_{k}\left(u-T_{h}(u)\right) d x \leq \\
\int_{\Omega} f T_{k}\left(u-T_{h}(u)\right) d x+\int_{\Omega} F . \nabla T_{k}\left(u-T_{h}(u)\right) d x+\left(d-\rho\left(u_{N e}\right)\right) T_{k}\left(u_{N e}-T_{h}\left(u_{N e}\right)\right) .
\end{array}\right.
$$

According to the fact that $\nabla T_{k}\left(u-T_{h}(u)\right)=\nabla u$ on $\{h \leq|u|<h+k\}$ and zero elsewhere, $\int_{\Omega}|u|^{p_{M}(x)-2} u T_{k}\left(u-T_{h}(u)\right) d x \geq 0$ and $\rho\left(u_{N e}\right) T_{k}\left(u_{N e}-T_{h}\left(u_{N e}\right)\right) \geq 0$, we deduce from (4.15) that

$$
\left\{\begin{array}{l}
\int_{F_{h, k}}\left(\sum_{i=1}^{N} a_{i}\left(x, \frac{\partial}{\partial x_{i}} u\right) \frac{\partial}{\partial x_{i}} T_{k}\left(u-T_{h}(u)\right)\right) d x \leq \\
k \int_{|u| \geq h}|f| d x+\int_{F_{h, k}}\left|\left(\frac{2}{C p_{m}^{-}}\right)^{\frac{1}{p_{m}^{-}}} F\right|\left|\left(\frac{C p_{m}^{-}}{2}\right)^{\frac{1}{p_{m}^{-}}} \nabla u\right| d x+k|d| .
\end{array}\right.
$$


Using (1.7) (in the left hand side of (4.16)), Young inequality (in the right hand side of(4.16)) and setting

we obtain

$$
c=\left(\frac{2}{C p_{m}^{-}}\right)^{\frac{\left(p_{m}^{\prime}\right)^{-}}{p_{m}^{-}}} \frac{p_{m}^{-}-1}{p_{m}^{-}},
$$

$$
\left\{\begin{array}{l}
\sum_{i=1}^{N} \int_{F_{h, k}}\left|\frac{\partial}{\partial x_{i}} u\right|^{p_{i}(x)} d x \leq \\
k \int_{|u| \geq h}|f| d x+c \int_{F_{h, k}}|F|^{\left(p^{\prime}\right)_{m}^{-}} d x+\frac{C}{2} \int_{F_{h, k}}|\nabla u|^{p_{m}^{-}} d x+k|d| .
\end{array}\right.
$$

From (4.14) and (4.17), we deduce

$$
\left\{\begin{array}{l}
C \int_{F_{h, k}}|\nabla u|^{p_{m}^{-}} d x \leq \\
k \int_{|u| \geq h}|f| d x+c \int_{F_{h, k}}|F|^{\left(p^{\prime}\right)_{m}^{-}} d x+\frac{C}{2} \int_{F_{h, k}}|\nabla u|^{p_{m}^{-}} d x+k|d|+N \text { meas }(\Omega) .
\end{array}\right.
$$

Therefore,

$$
\left\{\begin{array}{l}
\frac{C}{2} \int_{F_{h, k}}|\nabla u|^{p_{m}^{-}} d x \leq \\
k \int_{\{|u| \geq h\}}|f| d x+c \int_{F_{h, k}}|F|^{\left(p^{\prime}\right)_{m}^{-}} d x+k|d|+N \text { meas }(\Omega) .
\end{array}\right.
$$

Since $A_{2} \subset F_{h, k}$, we deduce from (4.18) that $\int_{A_{2}}|\nabla u|^{p_{m}^{-}} d x$ is bounded.

(2) $\lim _{h \rightarrow \infty}\left(\int_{A_{1}}|F|^{\left(p_{m}^{\prime}\right)^{-}} d x\right)^{\frac{1}{\left(p_{m}^{\prime}\right)^{-}}}=0$ (see $\left.[22]\right)$.

Now, it remains to prove that $\left(\int_{A_{1}}|\nabla u|^{p_{m}^{-}} d x\right)^{\frac{1}{p_{m}^{-}}}$is bounded with respect to $h$.

Since $A_{1} \subset F_{h-k, 2 k}$, we deduce from (4.18) that $\int_{A_{2}}|\nabla u|^{p_{m}^{-}} d x$ is bounded.

Remark 4.4. Similarly, we prove that if $v$ is an entropy solution of $P(\rho, f, d)$, then

$$
\lim _{h \rightarrow \infty} \int_{A_{2}^{\prime}} F . \nabla v d x \leq 0
$$

and

$$
\lim _{h \rightarrow \infty} \int_{A_{1}^{\prime}} F . \nabla v d x \leq 0
$$

Now, we have

$$
\begin{cases}A(h, k)=\int_{A_{0}}\left(\sum_{i=1}^{N}\left(a_{i}\left(x, \frac{\partial}{\partial x_{i}} u\right)-a_{i}\left(x, \frac{\partial}{\partial x_{i}} v\right)\right) \frac{\partial}{\partial x_{i}}(u-v)\right) d x & :=I_{0}(h, k) \\ +\int_{A_{1}}\left(\sum_{i=1}^{N} a_{i}\left(x, \frac{\partial}{\partial x_{i}} u\right) \frac{\partial}{\partial x_{i}} u\right) d x+\int_{A_{1}^{\prime}}\left(\sum_{i=1}^{N} a_{i}\left(x, \frac{\partial}{\partial x_{i}} v\right) \frac{\partial}{\partial x_{i}} v\right) d x & :=I_{1}(h, k) \\ +\int_{A_{2}}\left(\sum_{i=1}^{N} a_{i}\left(x, \frac{\partial}{\partial x_{i}} u\right) \frac{\partial}{\partial x_{i}}(u-v)\right) d x+\int_{A_{2}^{\prime}}\left(\sum_{i=1}^{N} a_{i}\left(x, \frac{\partial}{\partial x_{i}} v\right) \frac{\partial}{\partial x_{i}}(v-u)\right) d x & :=I_{2}(h, k) .\end{cases}
$$


The term $I_{1}(h, k)$ is non-negative since each term in $I_{1}(h, k)$ is non-negative.

For the term $I_{2}(h, k)$, as

$$
I_{2}(h, k)+\int_{A_{2}}\left(\sum_{i=1}^{N} a_{i}\left(x, \frac{\partial}{\partial x_{i}} u\right) \frac{\partial}{\partial x_{i}} v\right) d x+\int_{A_{2}^{\prime}}\left(\sum_{i=1}^{N} a_{i}\left(x, \frac{\partial}{\partial x_{i}} v\right) \frac{\partial}{\partial x_{i}} u\right) d x=I_{1}(h, k)
$$

so,

$$
I_{2}(h, k) \geq-\left(\int_{A_{2}}\left(\sum_{i=1}^{N} a_{i}\left(x, \frac{\partial}{\partial x_{i}} u\right) \frac{\partial}{\partial x_{i}} v\right) d x+\int_{A_{2}^{\prime}}\left(\sum_{i=1}^{N} a_{i}\left(x, \frac{\partial}{\partial x_{i}} v\right) \frac{\partial}{\partial x_{i}} u\right) d x\right) .
$$

Let us show that $-\left(\int_{A_{2}}\left(\sum_{i=1}^{N} a_{i}\left(x, \frac{\partial}{\partial x_{i}} u\right) \frac{\partial}{\partial x_{i}} v\right) d x\right)$ goes to 0 as $h \rightarrow \infty$.

We have

$$
\left\{\begin{array}{l}
\left|\int_{A_{2}}\left(\sum_{i=1}^{N} a_{i}\left(x, \frac{\partial}{\partial x_{i}} u\right) \frac{\partial}{\partial x_{i}}(v)\right) d x\right| \leq \\
C \sum_{i=1}^{N}\left(\left|j_{i}\right|_{p_{i}^{\prime}(.)}+\left|\frac{\partial u}{\partial x_{i}}\right|_{L^{p_{i}(.)}(\{h<|u| \leq h+k\})}^{p_{i}(x)-1}\right)\left|\frac{\partial v}{\partial x_{i}}\right|_{L^{p_{i}(\cdot)}(\{h-k<|v| \leq h\})}
\end{array} .\right.
$$

For all $i=1, \ldots N$, the quantity $\left(\left|j_{i}\right|_{p_{i}^{\prime}(.)}+\left|\frac{\partial u}{\partial x_{i}}\right|_{L^{p_{i}(.)}(\{h<|u| \leq h+k\})}^{p_{i}(x)-1}\right)$ is finite since $u=T_{h+k}(u) \in \mathcal{T}_{N e}^{1, \vec{p}(.)}(\Omega)$ and $j_{i} \in L^{p_{i}^{\prime}(.)}(\Omega)$; then by Lemma 3.8 , the last expression converges to zero as $h$ tends to infinity.

Similarly we can show that $-\left(\int_{A_{2}}\left(\sum_{i=1}^{N} a_{i}\left(x, \frac{\partial}{\partial x_{i}} v\right) \frac{\partial}{\partial x_{i}}(u)\right) d x\right)$ goes to 0 as $h \rightarrow \infty$, hence, we obtain

$$
\limsup _{h \rightarrow \infty} A(h, k) \geq \int_{[|u-v|<k]}\left[\sum_{i=1}^{N}\left(a_{i}\left(x, \frac{\partial}{\partial x_{i}} u\right)-a_{i}\left(x, \frac{\partial}{\partial x_{i}} v\right)\right) \frac{\partial}{\partial x_{i}}(u-v)\right] d x .
$$

By using the Lebesgue dominated convergence theorem, it yields that

$$
\lim _{h \rightarrow \infty} B(h, k)=\int_{\Omega}(b(u)-b(v)) T_{k}(u-v) d x \text { and } \lim _{h \rightarrow \infty} D(h, k)=0 .
$$

For $h$ large enough, we get

$$
\begin{gathered}
\lim _{h \rightarrow \infty} C(h, k)=\left(\rho\left(u_{N}\right)-\rho\left(v_{N}\right)\right) T_{k}\left(u_{N}-v_{N}\right) \text { and } \lim _{h \rightarrow \infty} E(h, k)=0 . \\
\left\{\begin{array}{l}
T(h, k)=\int_{A_{1}} F \cdot \nabla u d x+\int_{A_{1}^{\prime}} F \cdot \nabla v d x \\
+\int_{A_{2}} F \cdot \nabla(u-v) d x+\int_{A_{2}^{\prime}} F \cdot \nabla(v-u) d x .
\end{array}\right. \\
\left\{\begin{array}{l}
T(h, k)=\int_{A_{1}} F \cdot \nabla u d x+\int_{A_{1}^{\prime}} F \cdot \nabla v d x \\
+\int_{A_{2}} F \cdot \nabla u d x-\int_{A_{2}} F \cdot \nabla v d x+\int_{A_{2}^{\prime}} F \cdot \nabla v d x-\int_{A_{2}^{\prime}} F . \nabla u d x .
\end{array}\right.
\end{gathered}
$$


Using Assertion 4.3 and Remark 4.4, it is easy to see that $\lim _{h \rightarrow \infty}|T(h, k)|=0$. Letting $h$ go to $\infty$ in (4.11) and combining (4.20)-(4.21), we obtain

$$
\left\{\begin{array}{l}
\int_{[|u-v|<k]}\left[\sum_{i=1}^{N}\left(a_{i}\left(x, \frac{\partial}{\partial x_{i}} u\right)-a_{i}\left(x, \frac{\partial}{\partial x_{i}} v\right)\right) \frac{\partial}{\partial x_{i}}(u-v)\right] d x \\
+\int_{\Omega}(b(u)-b(v)) T_{k}(u-v) d x+\left(\rho\left(u_{N}\right)-\rho\left(v_{N}\right)\right) T_{k}\left(u_{N}-v_{N}\right) \leq 0 .
\end{array}\right.
$$

All the terms in the left hand side of (4.22) are non-negative so that we get $\forall k>0$,

$$
\int_{[|u-v|<k]}\left[\sum_{i=1}^{N}\left(a_{i}\left(x, \frac{\partial}{\partial x_{i}} u\right)-a_{i}\left(x, \frac{\partial}{\partial x_{i}} v\right)\right) \frac{\partial}{\partial x_{i}}(u-v)\right] d x=0
$$

and

$$
\left\{\begin{array}{l}
\int_{\Omega}(b(u)-b(v)) T_{k}(u-v) d x=0 \\
\left(\rho\left(u_{N}\right)-\rho\left(v_{N}\right)\right) T_{k}\left(u_{N}-v_{N}\right)=0 .
\end{array}\right.
$$

Relation (4.23) gives $\frac{\partial}{\partial x_{i}}(u-v)=0$ a.e. in $\Omega$; we deduce that there exists a constant $c$ such that $u-v=c$ a.e. in $\Omega$. According to $(4.24), b(u)=b(v)$. Since $b$ is invertible, we deduce that $u=v$ in $\Omega$ and so

$$
\left\{\begin{array}{l}
u=v \text { a.e. in } \Omega \\
\rho\left(u_{N}\right)=\rho\left(v_{N}\right)
\end{array}\right.
$$

which prove the uniqueness part. 


\section{References}

[1] A. Baalal, and M. Berghout, "The Dirichlet problems for nonlinear elliptic equations with variable exponent", Journal of Applied Analysis and Computation, vol. 9, no. 1, pp. 295-313, 2019.

[2] M. B. Benboubker, H. Hjiaj, and S. Ouaro, "Entropy solutions to nonlinear elliptic anisotropic problem with variable exponent", Journal of Applied Analysis and Computation, vol. 4, no. 3, pp. 245-270, 2014.

[3] M. Bendahmane, and K. H. Karlsen, "Anisotropic nonlinear elliptic systems with measure data and anisotropic harmonic maps into spheres", Electron. J. Differential Equations, no. 46, 30 pp, 2006.

[4] Ph. Bénilan, H. Brézis, and M. G. Crandall, "A semilinear equation in $L^{1}\left(\mathbb{R}^{N}\right)$ ". Ann. Scuola. Norm. Sup. Pisa, vol. 2, pp. 523-555, 1975.

[5] Ph. Bénilan, L. Boccardo, T. Gallouët, R. Gariepy, M. Pierre, and J. L. Vazquez, "An $L_{1}$ theory of existence and uniqueness of nonlinear elliptic equations", Ann Sc. Norm. Super. Pisa, vol. 22, no. 2, pp. 240-273, 1995.

[6] B. K. Bonzi, S. Ouaro, and F. D. Y. Zongo, "Entropy solution for nonlinear elliptic anisotropic homogeneous Neumann Problem", Int. J. Differ. Equ, Article ID 476781, 2013.

[7] M. M. Boureanu, and V. D. Radulescu, "Anisotropic Neumann problems in Sobolev spaces with variable exponent". Nonlinear Anal. TMA, vol. 75, no. 12, pp. 4471-4482, 2012.

[8] B. Koné, S. Ouaro and F. D. Y. Zongo, "Nonlinear elliptic anisotropic problem with Fourier boundary condition", Int. J. Evol. Equ, vol. 8, no 4, pp. 305-328, 2013.

[9] Y. Chen, S. Levine, and M. Rao, "Variable exponent, linear growth functionals in image restoration", SIAM J. Appl. Math, vol. 66, pp. 1383-1406, 2006.

[10] L. Diening, "Theoretical and Numerical Results for Electrorheological Fluids", PhD. thesis, University of Frieburg, Germany, 2002.

[11] L. Diening, "Riesz potential and Sobolev embeddings on generalized Lebesgue and Sobolev spaces $L^{p(.)}$ and $W^{1, p(.) ", ~ M a t h . ~ N a c h r, ~ v o l . ~ 268, ~ p p . ~ 31-43, ~} 2004$.

[12] Y. Ding, T. Ha-Duong, J. Giroire, and V. Mouma, "Modeling of single-phase flow for horizontal wells in a stratified medium", Computers and Fluids, vol. 33, pp. 715-727, 2004 . 
[13] X. Fan, and D. Zhao, "On the spaces $L^{p(\cdot)}(\Omega)$ and $W^{m, p(.)}(\Omega)$ ", J. Math. Anal. Appl., vol. 263, pp. 424-446, 2001.

[14] X. Fan, "Anisotropic variable exponent Sobolev spaces and $\vec{p}($.$) -Laplacian equations",$ Complex variables and Elliptic Equations. vol. 55, pp. 1-20, 2010.

[15] J. Giroire, T. Ha-Duong, and V. Moumas, "A non-linear and non-local boundary condition for a diffusion equation in petroleum engineering", Mathematical Methods in the Applied Sciences, vol. 28, no. 13, pp. 1527-1552, 2005.

[16] P. Halmos, Measure Theory, D. Van Nostrand Company, New York, 1950.

[17] T.C. Halsey, "Electrorheological fluids", Science, vol. 258, ed. 5083, pp. 761-766, 1992.

[18] H. Hudzik, "On generalized Orlicz-Sobolev space", Funct. Approximatio Comment. Math., vol. 4, pp. 37-51, 1976.

[19] I. Ibrango, and S. Ouaro, "Entropy solutions for nonlinear Dirichlet problems", Annals of the university of craiova, Mathematics and Computer Science Series, vol. 42, no. 2, pp. 347-364, 2015.

[20] I. Ibrango, and S. Ouaro, "Entropy solutions for nonlinear elliptic anisotropic problems with homogeneous Neumann boundary condition", Journal of Applied Analysis and Computation, vol. 6, no. 2, pp. 271-292, 2016.

[21] A. Kaboré, and S. Ouaro, "Nonlinear Elliptic anisotropic problem involving non local boundary conditions with variable exponent and graph data", Creative Mathematics, vol. 29 , no. 2 , pp. $145-152,2020$.

[22] I. Konaté, and S. Ouaro, "Good Radon measure for anisotropic problems with variable exponent" Electron. J. Diff Equ., vol. 2016, no. 221, pp. 1-19, 2016.

[23] O. Kovacik, and J. Rakosnik, "On spaces $L^{p(x)}$ and $W^{1, p(x)}$ ", Czech. Math. J., vol. 41, pp. 592-618, 1991.

[24] L. M. Kozhevnikova, "On solutions of elliptic equations with variable exponents and measure data in $\mathbb{R}^{n} ", 2019$. arXiv $: 1912.12432$.

[25] L. M. Kozhevnikova, "On solutions of anisotropic elliptic equations with variable exponent and measure data", Complex Variables and Elliptic Equations, vol. 65, no. 3, pp. 333-367, 2020.

[26] M. Mihailescu, and V. Radulescu, "A multiplicity result for a nonlinear degenerate problem arising in the theory of electrorheological fluids", Proc. R. Soc. Lond. Ser. A Math. Phys. Eng. Sci., vol. 462, pp. 2625-2641, 2006. 
[27] M. Mihailescu, and V. Radulescu, "On a nonhomogeneous quasilinear eigenvalue problem in Sobolev spaces with variable exponent", Proc. Amer. Math. Soc., vol. 135, pp. 2929-2937, 2007.

[28] M. Mihailescu, P. Pucci, and V. Radulescu, "Eigenvalue problems for anisotropic quasilinear elliptic equations with variable exponent”, J. Math. Anal. Appl., vol. 340, no. 1 , pp. $687-698,2008$.

[29] J. Musielak, Orlicz Spaces, and modular spaces, Lecture Notes in Mathematics, Springer, Berlin, 1983.

[30] H. Nakano, "Modulared semi-ordered linear spaces", Tokyo: Maruzen Co. Ltd, 1950.

[31] I. Nyanquini, S. Ouaro, and S. Safimba, "Entropy solution to nonlinear multivalued elliptic problem with variable exponents and measure data". Ann. Univ. Craiova ser. Mat. Inform., vol. 40, no.2, pp. 174-198, 2013.

[32] W. Orlicz, "Über konjugierte Exponentenfolgen", Studia Math., vol. 3, pp. 200-212, 1931.

[33] C. Pfeiffer, C. Mavroidis, Y. Bar-Cohen, and B. Dolgin, "Electrorheological fluid based force feedback device", in Proc. 1999 SPIE Telemanipulator and Telepresence Technologies VI Conf. (Boston, MA), vol. 3840, pp. 88-99, 1999.

[34] V. Radulescu, "Nonlinear elliptic equations with variable exponent: old and new", Nonlinear Anal., vol. 121, pp. 336-369, 2015.

[35] K.R. Rajagopal, and M. Ruzicka, "Mathematical modelling of electrorheological fluids", Continuum Mech. Thermodyn., vol. 13, pp. 59-78, 2001.

[36] M. Ruzicka, Electrorheological fluids: modelling and mathematical theory, Lecture Notes in Mathematics, Springer-Verlag, Berlin, 2000.

[37] M. Sanchon, and J. M. Urbano, "Entropy solutions for the $p(x)$-Laplace Equation", Trans. Amer. Math. Soc., vol. 361, no. 12, pp. 6387-6405, 2009.

[38] U. Sert, and K. Soltanov, "On the solvability of a class of nonlinear elliptic type equation with variable exponent", Journal of Applied Analysis and Computation, vol. 7, no. 3, pp. 1139-1160, 2019.

[39] M. Troisi, "Teoremi di inclusione per spazi di Sobolev non isotropi". Ric. Mat., vol. 18, pp. 3-24, 1969.

[40] I. Sharapudinov, "On the topology of the space $L^{p(t)}([0,1])$ ", Math. Zametki, vol. 26, pp. 613-632, 1978. 
[41] R. E. Showalter, Monotone operators in Banach space and nonlinear partial differential equations, Mathematical Surveys and Monographs, American Mathematical Society, Providence, RI, vol. 49, 1997.

[42] I.V. Tsenov, "Generalization of the problem of best approximation of a function in the space $L^{s ", ~ U c h . ~ Z a p . ~ D a g e s t a n ~ G o s . ~ U n i v ., ~ v o l . ~ 7, ~ p p . ~ 25-37, ~} 1961$.

[43] W. M. Winslow, "Induced Fibration of Suspensions", J. Applied Physics, vol. 20, pp. 1137-1140, 1949. 